04

\title{
Малоразмерный черенковский телескоп с полупроводниковыми фотодетекторами: полночастичное моделирование порога регистрации космических гамма-источников
}

\author{
(C) Е.Е. Холупенко, Д.В. Бадмаев, А.С. Антонов, А.А. Богданов, А.М. Красильщиков, Д.О. Кулешов, \\ Ю.В. Тубольцев, Е.М. Хилькевич, Ю.В. Чичагов
}

Физико-технический институт им. А.Ф. Иоффре РАН

194021 Санкт-Петербург, Россия

e-mail: eugene@astro.ioffe.ru

Поступило в Редакцию 25 июня 2021 г.

В окончательной редакции 9 августа 2021 г.

Принято к публикации 10 августа 2021 г.

\begin{abstract}
Выполнено массированное полночастичное моделирование физических процессов в атмосфере Земли, приводящих к генерации черенковского излучения широких атмосферных ливней от космических гаммаквантов и протонов космических лучей, а также моделирование процесса регистрации этого излучения малоразмерным черенковским телескопом с детекторной камерой, основанной на современных кремниевых фотоумножителях (SiPM) серии OnSemi MicroFJ. Расчеты выполнены для первичных частиц с энергиями от 0.3 до $30 \mathrm{TeV}$ и черенковского телескопа с площадью зеркала $\simeq 10 \mathrm{~m}^{2}$, подобного используемому в обсерватории TAIGA. Показано, что даже при использовании жестких критериев отбора, обеспечивающих высокое качество изображений регистрируемых событий в камере телескопа, пороговая энергия регистрации не превысит $0.8 \mathrm{TeV}$, что примерно в два раза ниже пороговой энергии регистрации $(\simeq 1.5 \mathrm{TeV})$ малоразмерного телескопа TAIGA-IACT с камерой на вакуумных фотоумножителях.
\end{abstract}

Ключевые слова: широкие атмосферные ливни, гамма-адронная сепарация (гамма-адронный отбор), черенковские гамма-телескопы, наблюдения в УФ диапазоне: 96.50.sd, 29.40.Ka, 41.60.Bq, 07.85.Nc, 07.05.Tp.

DOI: $10.21883 / J T F .2021 .12 .51759 .193-21$

\section{Введение}

Камеры черенковских гамма-телескопов, установленных на поверхности Земли, регистрируют вспышки черенковского излучения от релятивистских частиц, составляющих широкие атмосферные ливни (ШАЛ), инициированные первичными гамма-квантами, которые достигают Земли от ряда далеких космических объектов. Камеры большинства современных черенковских телескопов (MAGIC [1], VERITAS [2], H.E.S.S. [3]) состоят из высоковольтных вакуумных фотоумножителей (ФЭУ). Этот тип фотоэлементов обладает рядом недостатков, таких как сравнительно невысокая эффективность детектирования (как правило, не более $30 \%$ ), высокое энергопотребление (и высокое напряжение питания $>1000 \mathrm{~V}$ ), значительные габариты (ограничивающие возможность уменьшения как линейного, так и углового размеров пиксела при заданных конструкциях зеркала и монтировки) и возможность полного выхода из строя при избыточной засветке. Это заставляет специалистов искать новые типы фотоэлементов для камер, разрабатываемых в настоящее время черенковских гамма-телескопов нового (четвертого) поколения (например, СТА [4], ALEGRO [5]). В настоящее время наиболее подходящими кандидатами на роль таких фотоэлементов представляются полупроводниковые фотодетекторы - кремниевые фотоумножители (SiPM) - на основе которых со- здаются и успешно работают прототипы камер черенковских гамма-телескопов [6-11]. Такой выбор обусловлен тем, что в отличие от ФЭУ SiPM обладают сравнительно небольшими габаритами (3-10 mm), невысоким напряжением питания $(<100 \mathrm{~V})$, высокой эффективностью детектирования (photon detection efficiency, PDE), достигающей $\sim 50 \%$, и устойчивостью к избыточной засветке (что позволяет осуществлять наблюдения даже в лунные ночи [12], существенно увеличивая рабочий цикл).

$\mathrm{C}$ целью отработки технологий создания камер черенковских гамма-телескопов четвертого поколения (в частности, в рамках проекта ALEGRO [5,13]) и повышения эффективности работы существующих черенковских гамма-обсерваторий в ФТИ им. А.Ф. Иоффе производится разработка детекторного кластера на основе фотодекторов SiPM OnSemi MicroFJ-60035 для действующего черенковского телескопа TAIGA-IACT [14] (НИИЯФ МГУ им. М.В. Ломоносова, Иркутский государственный университет), расположенного в Тункинской долине (республика Бурятия). Кремниевые фотоумножители OnSemi MicroFJ-60035 обладают высокой эффективностью детектирования (до $\simeq 50 \%$ в максимуме [15], см. рис. 1), что значительно превышает эффективность вакуумных фотоумножителей ХР1911 ( $26 \%$ в максимуме [16], см. рис. 1), используемых в нынешней конфигурации камеры TAIGA-IACT. 


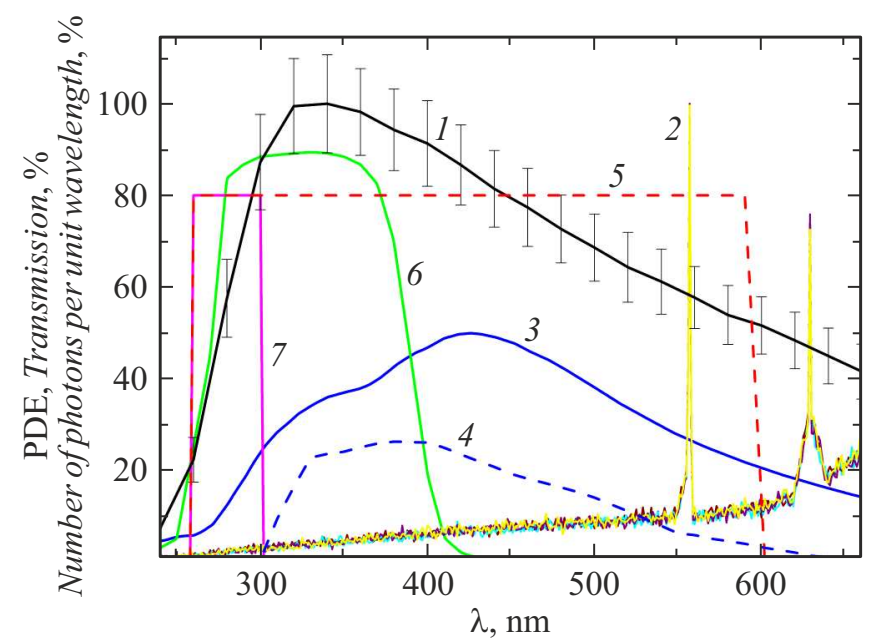

Рис. 1. Зависимости профилей от длины волны $\lambda: 1-$ средний спектр черенковского излучения ШАЛ от гамма-кванта с энегией $1 \mathrm{TeV}$, нормированный на $100 \%$ в максимуме (на длине волны $\simeq 330 \mathrm{~nm}), N_{\mathrm{Ch}}(\lambda) / N_{\mathrm{Ch}}(330 \mathrm{~nm})$ - черная кривая; 2 - примеры четырех конкретных реализаций спектра фона ночного неба, полученных методом Монте-Карло на основании данных $[18,19,40,41]$, нормированные на $100 \%$ для максимального (из приведенных реализаций) значения на длине волны $\simeq 557 \mathrm{~nm}, I_{\lambda} / I_{557}^{\max }$ - частично перекрывающиеся желтая, фиолетовая, голубая и бордовая кривые (в online версии); 3 - эффективность детектирования SiPM фотоумножителя OnSemi MicroFJ-60035 по данным [15], $\operatorname{PDE}(\lambda) ; 4$ - эффективность вакуумного фотоумножителя ХР1911 по данным $[16,69] ; 5-$ коэффициент пропускания $F_{\text {opt }}(\lambda)$ модельного фильтра основного диапазона $260-600 \mathrm{~nm} ; 6-$ коэффициент пропускания $F_{\mathrm{ZWB}}(\lambda)$ фильтра ZWB3 по данным [22]; 7 - коэффициент пропускания $F_{\mathrm{MUV}}(\lambda)$ модельного MUV-фильтра $260-300 \mathrm{~nm}$.

Помимо использования SiPM модернизация камеры телескопа TAIGA-IACT предполагает осуществление наблюдений с использованием сменных фильтров различных типов [17]. Фильтр основного рабочего диапазона ( 260-600 nm, рис. 1) необходим, чтобы ограничить общую чувствительность телескопа со стороны больших длин волн $\lambda$, поскольку начиная $\mathrm{c} \simeq 620 \mathrm{~nm} \mathrm{ин-}$ тенсивность фона ночного неба, являющегося основным фактором генерации шумового сигнала в камере, резко возрастает из-за излучения в атмосфере Земли атомарного кислорода в линиях 630 и $636 \mathrm{~nm}$ и гидроксила $(\mathrm{OH})$ в полосах Мейнеля $[18,19]$, а интенсивность черенковского излучения падает пропорционально $\lambda^{-2}$. Таким образом, в диапазоне длин волн $\lambda \gtrsim 620 \mathrm{~nm}$ условия регистрации радикально ухудшаются по сравнению с диапазоном $\lambda \lesssim 600 \mathrm{~nm}$. При этом детектор OnSemi MicroFJ-60035 сохраняет в диапазоне 620-800 nm значительную эффективность детектирования $(18-5 \%)$, которая не может быть подавлена только с помощью регулировки параметров самого детектора без ухудшения чувствительности в основном рабочем диапазоне. Фильтр ближнего УФ-диапазона (NUV) еще более значительно подавляет шумовой сигнал в каме- ре телескопа, вызванный оптическим фоном ночного неба и лунной засветкой, и при этом в значительной мере сохраняет полезный сигнал от ШАЛ (рис. 1), что позволяет увеличить длительность рабочего цикла черенковского телескопа [20-24]. В качестве конкретного типа NUV-фильтра в рамках настоящих расчетов был выбран фильтр ZWB3 ( 270-400 nm, рис. 1). Использование фильтров среднего УФ-диапазона (MUV) позволяет повысить эффективность стандартных методов определения типа первичной частицы (так называемой „гамма-адронной сепарации“ с использованием параметров Хилласа [25]) посредством осуществления грубой спектрометрии черенковских вспышек ШАЛ измерения доли УФ-излучения в общем количестве черенковского излучения ШАЛ [26-29].

Для того чтобы количественно оценить эффективность предстоящей модернизации камеры телескопа TAIGA-IACT и рассчитать характеристики телескопа (прежде всего, пороговую энергию регистрации), необходимо провести численное моделирование процессов генерации излучения ШАЛ и его регистрации в камере телескопа. Моделирование некоторых режимов работы телескопа TAIGA-IACT было проведено в работе [17] на основе полуаналитической модели формирования и распространения ШАЛ и аналитической модели телескопа. Хотя эти модели являются наглядными и позволяют достаточно быстро получать предварительные оценки параметров эффективности телескопа для широкого диапазона параметров, они обладают рядом существенных недостатков: отсутствием полноты статистического описания (выполняется оценка только некоторых средних значений рассматриваемых величин), использованием большого количества приближений и экстраполяций, приближенными формулировками условий выработки триггера для регистрации события и т.д. Вследствие сказанного, результаты расчетов [17] следует рассматривать как оценочные и требующие дополнительного обоснования и уточнения.

Настоящая работа посвящена полночастичному моделированию методом Монте-Карло различных режимов работы малоразмерного черенковского телескопа с камерой на SiPM, близкого по исходным геометрическим параметрам (таким, как площадь зеркала, фокусное расстояние, размер детекторных пикселей и т.п.) к телескопу TAIGA-IACT. Моделирование черенковского излучения ШАЛ осуществлялось с помощью специализированного пакета CORSIKA [30], моделирование фона ночного неба, а также моделирование процессов регистрации череновских фотонов в телескопе осуществлялось с помощью оригинального пакета кодов TAIGA Soft (также использующего методы МонтеКарло). Поскольку многочастичные расчеты требуют значительных вычислительных мощностей, охватить ими все пространство параметров регистрируемых событий не представляется возможным, однако по сравнению с полуаналитическими вычислениями, выполненными ранее, такие расчеты позволяют получить значительно 
более полные и обоснованные оценки характеристик телескопа, а общее согласие результатов полночастичного моделирования с аналитическими оценками говорит об адекватности обоих подходов поставленным задачам.

\section{1. Моделирование черенковского излучения ШАЛ}

Формирование и распространение черенковского излучения ШАЛ моделировалось с помощью специализированного пакета кодов CORSIKA v. 7.7400 [30]. В настоящее время этот пакет широко используется для моделирования ШАЛ (согласно базе данных NASA ADS, количество цитирований базовой работы [30] превышает 1000), а его результаты считаются эталонными.

Моделирование осуществлялось при следующих начальных условиях (исходных данных): тип первичной частицы - гамма-кванты и протоны; энергии первичных частиц $-0.3162,0.5,0.7,1.0,3.162,10.0$ и $31.62 \mathrm{TeV}$; падение - вертикальное (зенитный угол равен нулю); прицельный параметр первичных частиц (при вертикальном падении совпадает с расстоянием от телескопа до оси ШАЛ, вызванного первичной частицей) составляет $120 \mathrm{~m}$; величина магнитного поля $(\mathrm{H}, \mathrm{Z})=(18.5,57.7)$ $\mu \mathrm{T}$ [31]; высота наблюдения - $675 \mathrm{~m}$ над уровнем моря [32] (глубина атмосферы $\simeq 950-960 \mathrm{~g} / \mathrm{cm}^{3}$ ); используемая модель атмосферы - N3 (зимняя среднеширотная [33]); рассматриваемый диапазон длин волн фотонов черенковских фотонов 200-700 nm; размер пучка (параметр CERSIZ — bunch size) составлял 1 фотон. Последнее обстоятельство является довольно важным и требует пояснения. Часто при моделировании черенковского излучения ШАЛ от частиц высоких энергий $(E>\mathrm{TeV})$ для уменьшения требований к вычислительным ресурсам фотоны этого излучения с близкими значениями вектора распространения объединяют в пучки (bunch), каждый из которых впоследствии рассматривается и обрабатывается как единый объект. Поскольку фотоны одного пучка могут иметь весьма различные энергии, то объединение в пучки приводит фактически к потере информации о длинах волн этих фотонов. По этой причине при расчетах не рекомендуется одновременно производить объединение в пучки и учет атмосферного поглощения. Однако неучет атмосферного поглощения может привести к значительным отличиям в прогнозируемом количестве черенковских фотонов. Чтобы результаты сохраняли корректность при использовании объединения в пучки, исследуемый диапазон стараются ограничить областями длин волн, в которых атмосферное поглощение не является значительным, что, в свою очередь, не всегда соответствует исходной постановке задачи. В рассматриваемой задаче исследовано черенковское излучение, в частности, на длинах волн 260-400 nm, где атмосферное поглощение необходимо учитывать, поскольку проект модернизации камеры предполагает использование соответствующих фильтров для подавления фона ночного неба (фильтр типа ZWB3 охватывает диапазон $270-400 \mathrm{~nm})$ и осуществления грубой спектрометрии (фильтр с полосой пропускания 260-300 nm типа MUV [model/middle UV]). Кроме того, объединение фотонов в пучки требует неестественной адаптации модели телескопа, делающей ее недостаточно реалистичной; при этом приходилось бы разыгрывать не вероятность прохождения фотона через тот или иной узел оптического тракта телескопа, а фактически количество фотонов пучка, которым это прохождение удалось, что при небольших размерах пучка $(\lesssim 5)$ вляется довольно грубым приближением даже с чисто статистической точки зрения. Некорректность подобного подхода становится еще более явной, если учесть факт потери информации о длинах волн фотонов при объединении в пучок, что, в частности, приводит к необходимости использования некоторых средних по спектру коэффициентов передачи узлов оптического тракта и эффективности детектирования фотоэлементов, в то время как их реальные дифференциальные (зависящие от длины волны) значения могут на порядок отличаться от подобного среднего значения. С другой стороны, если размер пучка будет очень большим (> 10), это может привести к неоправданному искажению распределения черенковских фотонов ШАЛ и, как следствие, чрезмерному огрублению „снимка“ моделируемой черенковской вспышки. Таким образом, имеются веские основания для проведения моделирования черенковского излучения ШАЛ без использования приближения объединения фотонов в пучки (т.е. при размере пучка - единица), несмотря на то, что это требует значительно больших вычислительных ресурсов.

Значительный объем требуемых вычислительных ресурсов (как объемов используемой оперативной и постоянной памяти, так и процессорного времени) приводит к тому, что на первом (предварительном) этапе моделирования целесообразно провести расчеты для конкретного значения прицельного параметра падающих частиц, т.е. для конкретного значения расстояния от зеркала телескопа до оси ШАЛ. В настоящей работе для оценки характеристик регистрируемого сигнала была выбрана позиция телескопа на характерном расстоянии 120 m от оси ШАЛ. Помимо необходимости сравнения с результатами работы [17] (где также было использовано такое значение этого параметра), это сделано по следующим соображениям. С одной стороны, как уже было отмечено в [17], на расстояниях $r$ свыше 120-140 m от оси ШАЛ поверхностная плотность черенковских фотонов $\rho(r, E),\left[\mathrm{m}^{-2}\right]$, которая в значительной степени определяет величину полезного сигнала в камере телескопа, убывает по закону $\sim r^{-2}$ (рис. 2), что приводит к значительному уменьшению вероятности регистрации полезных событий на расстояниях более $150 \mathrm{~m}$ от оси ШАЛ для энергий первичных гамма-квантов, близких к пороговой энергии наблюдений (см., например, [34]). С другой стороны, поскольку поток первичных гаммаквантов от заданного космического источника однороден 


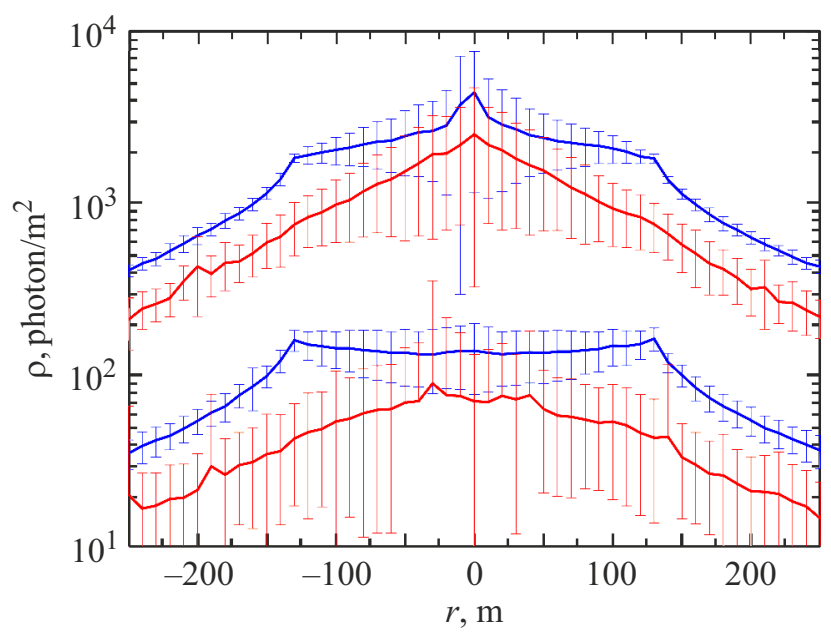

Рис. 2. Пространственные распределения (поверхностные плотности, $\rho, \mathrm{m}^{-2}$ ) черенковского излучения как функции расстояния до оси ШАЛ, рассчитанные с помощью многочастичного симулятора ШАЛ CORSIKA для энергий $1 \mathrm{TeV}$ (нижняя пара кривых) и $10 \mathrm{TeV}$ (верхняя пара кривых) и различных типов первичных частиц: гамма-квантов (синие кривые) и протонов космических лучей (красные кривые (в online версии)).

относительно смещения телескопа в плоскости, перпендикулярной направлению на источник, относительная вероятность того, что пришедший гамма-квант имеет прицельный параметр в интервале $[r ; r+d r]$ пропорциональна $r$. Скорость счета в интервале $[r ; r+d r]$ пропорциональна произведению вероятности регистрации и относительной вероятности того, что пришедший гамма-квант имеет прицельный параметр в интервале $[r ; r+d r]$. Соответственно основной вклад в скорость счета на энергиях, близких к пороговой, будут вносить гамма-кванты с прицельными параметрами около 120-150 m. Таким образом, выбранное расстояние от телескопа до оси ШАЛ $(120 \mathrm{~m})$, близко к некоторому среднему значению прицельных параметров наблюдаемых гамма-квантов, что позволяет достаточно корректно оценить пороговую энергию наблюдений.

Моделирование черековского излучения ШАЛ с кодом CORSIKA позволяет получить список фотонов, достигающих поверхности Земли, в следующем формате: $\left\{N_{p h}, X_{p h}, Y_{p h}, u, v, t, h,-\lambda\right\}$, где $N_{p h}-$ количество фотонов в пучке, $X_{p h}, Y_{p h},[\mathrm{~cm}]$ - координаты точки на поверхности, которой достигает пучок фотонов, $u, v-$ направляющие косинусы относительно горизонтальных осей $X, Y, t,[\mathrm{~ns}]$ - время с момента первого взаимодействия, $h,[\mathrm{~cm}]$ - высота генерации пучка, $\lambda,[\mathrm{nm}]-$ длина волны. При этом указанный список содержит информацию о всех событиях серии. Для дальнейшей обработки этих данных с помощью оригинального кода из пакета TAIGA Soft, моделирующего транспорт фотонов в телескопе и их регистрацию, указанный список преобразуется в набор файлов, содержащих данные в следую- щем формате: $\left\{\lambda, X_{p h}, Y_{p h}, u, v, w\right\}$ (где $w$ - направляющий косинус относительно вертикальной оси $Z$ ) с помощью промежуточного кода преобразования форматов. Разница в форматах возникает как по историческим, так и по практическим причинам. Пакет TAIGA Soft, предназначенный для моделирования телескопа TAIGA-IACT, является развитием пакета ALEGRO Soft, предназначенного для моделирования телескопа ALEGRO $[5,13]$ с большим зеркалом $(\sim 30 \mathrm{~m})$ и низкой пороговой энергией наблюдений $(\sim 5 \mathrm{GeV})$. Оба пакета содержат оригинальный код [35,36] (использующий последний из указанных форматов) на основе пакета библиотек GEANT4 [37,38] (и ссылки там же) для моделирования ШАЛ, что в принципе позволяет осуществлять полный цикл моделирования работы телескопа без привлечения стороннего программного обеспечения. Таким образом, для сопряжения пакета TAIGA Soft с кодом CORSIKA необходим дополнительный код преобразования форматов. Практическая выгода от использования оригинального формата состоит в том, что файлы, содержащие информацию об отдельных событиях, имеют приемлемый для обработки и передачи объем даже при очень больших энергиях первичных частиц и больших площадях наблюдения (когда число исследуемых черенковских фотонов очень велико, $\left.>10^{7}\right)$, в отличие от файла в формате CORSIKA, содержащего информацию о всей серии событий, и имеющего очень большой объем, что может создавать определенные сложности при его обработке и передаче по сети.

Важно отметить, что в процессе преобразования формата модельных данных используется следующая процедура, имеющая физический смысл. При использовании размера пучка (параметр CERSIZ) равного единице, количество фотонов в пучке $N_{p h}$ может принимать дробные значения меньше единицы и должно быть интерпретировано как вероятность достижения заданной точки соответствующим фотоном [39]. Таким образом, в процессе работы кода, преобразующего формат, для верификации фотона осуществляется розыгрыш (на основе равномерного распределения в интервале $[0 ; 1])$ параметра достижения поверхности наблюдения. Если этот параметр не превышает значение $N_{p h}$, то считается, что данный фотон достиг поверхности наблюдения и его параметры записываются в файл результатов.

Полученные в результате работы кода по преобразованию формата данные являются исходными для работы кода, непосредственно моделирующего работу оптического тракта телескопа TAIGA-IACT (разд. 3).

\section{2. Моделирование фона ночного неба}

Моделирование фона ночного неба было выполнено c помощью оригинального кода из пакета TAIGA Soft. В исследуемом диапазоне длин волн фон ночного неба формируется за счет множества различных вкладов [18], таких как свет звезд (прямой и рассеянный межзвезд- 
ной пылью), зодиакальный свет, свечение атмосферы (рекомбинация, люминесценция, хемилюминесценция), свет, рассеянный тропосферой и т.п. Наличие большого количества перекрывающихся линий и диффузного излучения с континуальным спектром приводит к образованию сложного квазинепрерывного спектра, который подвержен значительным временным и пространственным вариациям (как случайным флуктуациям, так и систематическим вариациям). Моделирование частных реализаций этого спектра методом Монте-Карло требует задания определенных исходных данных - среднего спектра фона ночного неба. Функциональная зависимость сглаженного среднего спектра фонового излучения $\bar{I}_{\lambda}$ от длины волны $\lambda$ была получена на основании данных из работы [18]. Дополнительными спектральными деталями, которые были учтены при моделировании фона ночного неба, являются линии атомарного кислорода $557.7 \mathrm{~nm}$ и дублет ${ }^{1} 630.2$ и $636.4 \mathrm{~nm}$ (приближенно смоделирован как одна линия со значительным уширением основания) - наиболее яркие эмиссионные детали ночной атмосферы в диапазоне 200-700 nm [19,40,41]. Повышательный тренд в диапазоне $2640 \mathrm{~nm}$ имитирует излучение атмосферного гидроксила $\mathrm{OH}$ в полосах Мейнеля $[18,19]$. Общая нормировка спектра составила $I=\int I_{\lambda} d \lambda=3 \cdot 10^{12} \mathrm{phot} \cdot \mathrm{m}^{-2} \cdot \mathrm{s}^{-1} \cdot \mathrm{st}^{-1}$ в диапазоне длин волн 300-600 nm на основании данных, предоставленных научной группой TAIGA-IACT [14], что хорошо согласуется с данными работы [42] для широкоугольного детектора (с полем зрения $\gg 1^{0}$ ), размещенного в средних широтах, при осуществлении наблюдений в направлении зенита и вне галактической плоскости.

Моделирование фона ночного неба состоит из следующих этапов:

1) Вычисление общего количества фоновых фотонов, падающих на заданную квадратную площадку:

$$
N_{\text {tot }}=I a^{2} \Delta t 2 \pi\left(1-\cos \theta_{\text {lim }}\right)
$$

где $a=5 \mathrm{~m}$ - сторона площадки (охватывающей телескоп), за время $\Delta t=15 \mathrm{~ns}$ с направлений, ограниченных зенитным углом $\theta_{\lim }=5.73^{\circ}(0.1 \mathrm{rad})$, и охватывающих все поле зрения телескопа TAIGA-IACT.

2) Определение (для каждого фотона) направляющего косинуса относительно оси $Z$, согласно формуле

$$
w=\cos \theta=1-\left(1-\cos \theta_{\lim }\right) \xi_{1},
$$

где $\theta-$ зенитный угол фотона, $\xi_{1}$ - случайная величина, равномерно распределенная в интервале $[0 ; 1]$.

\footnotetext{
${ }^{1}$ Следует отметить, что включение данного дублета в настоящее моделирование не является принципиальным, поскольку предполагается, что модернизированная камера будет эксплуатироваться с применением фильтров, и длинноволновая граница исследуемого диапазона составит $\sim 600 \mathrm{~nm}$. Таким образом, учет данной спектральной детали выполнен для большей полноты модели и является, скорее, заделом для дальнейших исследований, в которых могут быть изучены режимы наблюдения с фильтрами, обладающими пропусканием в диапазоне длин волн выше $600 \mathrm{~nm}$.
}

3) Определение (для каждого фотона) азимутального угла $\phi$ и вычисление направляющих косинусов относительно осей $X, Y$ согласно формулам

$$
\phi=\pi \xi_{2}, u=\cos \phi \sin \theta, v=\sin \phi \sin \theta
$$

где $\xi_{2}-$ случайная величина, равномерно распределенная в интервале $[-1 ; 1]$.

4) Определение (для каждого фотона) координат точки падения $X_{\mathrm{ph}}, Y_{\mathrm{ph}}$, согласно формулам

$$
X_{\mathrm{ph}}=a \xi_{3} / 2, \quad Y_{\mathrm{ph}}=a \xi_{4} / 2,
$$

где $\xi_{3}, \xi_{4}-$ случайные величины, равномерно распределенные в интервале $[-1 ; 1]$.

5) Определение (для каждого фотона) длины волны, согласно формуле $\lambda=\Lambda\left(\xi_{5}\right)$, где $\xi_{5}-$ случайная величина, равномерно распределенная в интервале [0;1], а функция $\Lambda(\xi)$ неявно определена следующими соотношениями:

$$
I_{\text {tot }} \xi=\int_{0}^{\Lambda} I_{\lambda} d \lambda, I_{\text {tot }}=\int_{0}^{\infty} I_{\lambda} d \lambda .
$$

Моделирование фона ночного неба методом МонтеКарло позволяет получить как списки фотонных событий, соответствующие отдельным реализациям „кадрам“ с экспозицией $\Delta t$, так и спектральные распределения фона для конкретных реализаций.

Примеры конкретных реализаций спектра фона ночного неба приведены на рис. 1, где показаны величины относительных спектральных распределений $I_{\lambda} / I_{557}^{\max }$, где $I_{557}^{\max }$ - максимальное из приведенных реализаций значение спектральной плотности на длине волны $\simeq 557 \mathrm{~nm}$ (т.е. одно из этих распределений нормировано на $100 \%$ на длине волны $\simeq 557 \mathrm{~nm}$, а остальные имеют в максимуме меньшие значения).

Модельные данные о фоне ночного неба и модельные данные о черенковском излучении ШАЛ (разд. 1), являются исходными данными для непосредственного моделирования транспорта фотонов в оптическом тракте телескопа TAIGA-IACT (разд. 3).

\section{3. Моделирование транспорта фотонов в телескопе и регистрации событий в детекторной камере}

Моделирование транспорта фотонов и регистрации событий в телескопе состоит из следующих этапов:

1) Проверка условия, что фотон попадает в поле зрения телескопа: $\theta \leq \mathrm{FoV} / 2$, где $\mathrm{FoV}=9.72^{0}$;

2) Проверка условия, что фотон падает на зеркало телескопа: $R_{\mathrm{ph}} \leq R_{T}^{\mathrm{eff}}, \quad$ где $R_{T}^{\mathrm{eff}}=1.75 \mathrm{~m}-$ эффективный радиус зеркала телескопа, $R_{\mathrm{ph}}=\left(\left(X_{\mathrm{ph}}-X_{T}\right)^{2}+\left(Y_{\mathrm{ph}}-Y_{T}\right)^{2}\right)^{1 / 2}-$ расстояние от точки падения фотона до оси телескопа, $X_{T}, Y_{T}-$ 
координаты точки стояния телескопа в системе отсчета, связанной с осью ШАЛ (т.е. ось ШАЛ проходит через точку (0;0)). Используемое значение радиуса зеркала $R_{T}^{\mathrm{eff}}$ выбрано исходя из условия $S_{m}=\pi\left(R_{T}^{\mathrm{eff}}\right)^{2} \simeq 9.6 \mathrm{~m}^{2}$ и отличается от указанного в работах по TAIGA-IACT значения $R_{T} \simeq 2.15 \mathrm{~m}$, поскольку реальное зеркало состоит из 34 сегментов диаметром $60 \mathrm{~m}$ каждый [43]. При этом с одной стороны, учесть сегментную структуру достаточно сложно, но с другой стороны, использование эффективного радиуса представляется достаточно адекватным приближением для данной модели;

3) Вычисление координат фотона в фокальной плоскости по следующим формулам (см. например, $[44,45])$ :

$$
x_{\mathrm{ph}}=K \theta_{X}, \quad y_{\mathrm{ph}}=K \theta_{Y},
$$

где $K \simeq 8 \mathrm{~cm} / \operatorname{arcdeg}, \theta_{X} \simeq \theta \cos \phi, \theta_{Y} \simeq \theta \sin \phi$;

4) Розыгрыш исхода отражения фотона от зеркала $\xi_{6} \leq V_{m}(\lambda)$, где $\xi_{6}-$ случайная величина, равномерно распределенная в интервале $[0 ; 1], V_{m}(\lambda)$ - коэффициент отражения зеркала. В настоящих расчетах принято не зависящее от длины волны $\lambda$ округленное значение $V_{m}=0.9$, близкое к типичным значениям коэффициентов отражения алюминиевых зеркал как телескопов оптического диапазона (например, [46]), так и черенковских телескопов (см., например, [47]). По данным [16] коэффициент отражения зеркал второго и третьего телескопов TAIGA-IACT составит > 85\%.

Следует отметить, что текущая модель телескопа позволяет учесть поворот зеркала в любом направлении, но это в настоящих расчетах не используется, поскольку исследуются события, вызванные вертикально падающими первичными частицами и предполагается, что телескоп направлен в зенит (хотя следует отметить, что полученные результаты в целом должны быть справедливы и для малых зенитных углов $\left.\lesssim 10^{\circ}\right)$. Поэтому процедура поворота системы координат телескопа в настоящей работе не приводится.

В результате моделирования транспорта фотонов в телескопе создается список событий в формате $\left\{x_{\mathrm{ph}}, y_{\mathrm{ph}}, \lambda\right\}$ (каждая строка соответствует отдельному фотону). Эти данные используются для дальшейшего моделирования регистрации событий в детекторной камере.

Моделирование камеры состоит из следующих этапов:

1) Розыгрыш исхода прохождения фотона через защитное покрытие камеры (в текущем варианте - плита из оргстекла толщиной $15 \mathrm{~mm}$, в планируемом варианте модернизации - плита, содержащая окна из кварцевого стекла с высоким коэффициентом пропускания в УФдиапазоне) $\xi_{7} \leq V_{g}(\lambda)$, где $\xi_{7}-$ случайная величина, равномерно распределенная в интервале $[0 ; 1], V_{g}(\lambda)-$ коэффициент пропускания защитного покрытия. В настоящих расчетах принято не зависящее от длины волны $\lambda$ округленное значение коэффициента пропускания $V_{g}=0.9$, не превышающее типичных значений коэффициентов пропускания кварцевых стекол (например, КУ-1, КУ-2, КУВИ [48]).
2) Розыгрыш исхода прохождения фотона через фильтр $\xi_{8} \leq F_{F}(\lambda)$, где $\xi_{8}-$ случайная величина, равномерно распределенная в интервале $[0 ; 1], F_{F}(\lambda)-$ коэффициент пропускания конкретного рассматриваемого фильтра (индекс $F$ принимает значения „орt“ [основной диапазон], „ZWB3““ или „MUV“). Зависимости коэффициентов пропускания фильтров от длины волны $\lambda$ приведены на рис. 1.

3) Розыгрыш исхода прохождения фотона через оптический концентратор (конус Уинстона) $\xi_{9} \leq V_{W}(\lambda)$, где $\xi_{9}$ - случайная величина, равномерно распределенная в интервале $[0 ; 1], V_{W}(\lambda)-$ коэффициент трансмиссии. В настоящих расчетах значение этого коэффициента принято не зависящим от длины волны $\lambda$ и составляет $V_{W}=0.75$ на основании оценки минимального значения коэффициента трансмиссии по данным работы [49] для конусов Уинстона с конфигурацией, сходной с планируемой к использованию. Моделирование конусов Уинстона, выполненное в работе [50] в рамках того же проекта модернизации камеры телескопа TAIGA-IACT, что и настоящая работа, ${ }^{2}$ показывает, что при использовании шестиугольных входных и выходных окон может быть достигнут коэффициент трансмиссии $\simeq 72.6 \%$, что согласуется с использованным значением с относительной точостью лучше 4\%. Некоторое снижение коэффициента трасмиссии обусловлено несоответствием формы выходного окна (шестиугольник) и формы активной области фотоэлементов (квадрат), что приводит к неполному покрытию площади выхдного окна и некоторым дополнительным потерям фотонов по сравнению со стандартной компоновкой (когда входное окно ФЭУ полностью покрывает площадь выходного окна конуса Уинстона).

4) Розыгрыш исхода регистрации фотона фотоэлементом (кремниевым фотоумножителем) $\xi_{10} \leq \operatorname{PDE}(\lambda)$, где $\xi_{10}$ - случайная величина, равномерно распределенная в интервале $[0 ; 1], \operatorname{PDE}(\lambda)$ - эффективность детектирования SiPM OnSemi MicroFJ-60035 [15], зависимость которой от длины волны $\lambda$ приведена на рис. 1 .

5) Определение номера конкретного пикселя детекторной камеры, в который попал фотон, посредством сопоставления координат фотона в фокальной плоскости $x_{p h}, y_{p h}$ и ячеек автоматически сгенерированной гексагональной сетки, которые соответствуют отдельным пикселям.

6) Запись результата в пиксельную карту - двумерный массив, $\left(Q_{F}^{S}\right)_{i k}$ [ph.e./pixel], содержащий данные о количестве фотоэлектронов, сгенерированных в конкретных пикселях (пронумерованных индексами $i, k$ ) за время регистрации события. Верхний индекс $S$ (означает

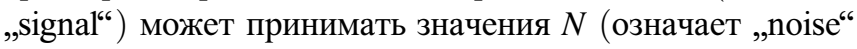

\footnotetext{
${ }^{2}$ Ввиду ограниченности вычислительных ресурсов моделирование различных узлов телескопа приходится выполнять параллельно (одновременно). Это не позволило сразу использовать в настоящих расчетах наиболее подходящие и обоснованные значения параметров узлов планируемой камеры, соответствующие именно поставленной задаче, а заставило прибегнуть к оценочным значениям, основанным на данных работ других научных групп по соответствующей тематике.
} 
и соответствует шумовому сигналу, вызванному фоном ночного неба), $g$ (означает ,gатmа“ и соответствует полезному сигналу, вызванному событиями от гаммаквантов), $p$ (означает „proton“ и соответствует полезному сигналу, вызванному событиями от протонов), нижний индекс $F$ означает „filter“ и может принимать значения opt (для фильтра основного рабочего диапазона), ZWB (для фильтра ZWB3) и MUV (для модельного фильтра MUV).

В результате моделирования регистрации событий в детекторной камере создается список событий в виде двумерных массивов - попиксельных карт $\left(Q_{F}^{S}\right)_{i k}$. Эти данные используются для дальшейшего анализа и выделения полезных гамма-событий из фона ночного неба и фона, создаваемого частицами космических лучей.

Следует отметить, что финальный этап моделирования работы детекторной камеры - формирование суммарной попиксельной карты (сигнал + шум) 一 также осуществляется в коде, используемом для анализа полученных снимков. Это делается для удобства моделирования и оценки параметров шума в отсутствие полезного сигнала, а также связано с необходимостью визуализации изображения черенковской вспышки ШАЛ и изображения фона ночного неба как вместе, так и по отдельности для определения и демонстрации эффективности алгоритма очистки изображений (разд. 4.2).

\section{4. Идентификация и обработка полезного сигнала}

Получение данных о ШАЛ и измерение характеристик энергичных первичных частиц, создающих ШАЛ в земной атмосфере, с помощью черенковского гаммателескопа подразумевает два основных этапа обработки и анализа сигнала: (i) быстрая первичная (front-end) обработка данных на аппаратном уровне с целью идентификации сигнала ШАЛ на фоне излучения ночного неба и приборном фоне и (ii) медленная вторичная (back-end) обработка идентифицированных событий на программном уровне с целью определения характеристик первичных частиц (гамма-квантов или космических лучей) на основе параметров Хилласа [25].

\section{1. Условия формирования триггера на запись данных}

Условия выдачи триггера на запись данных в постоянную память включают в себя следующие:

1) Выбор (определение) порогового значения сигнала, при превышении которого осуществляется запись. В настоящей работе это значение определено как $Q_{F}^{\text {th }}=Q_{F}^{N}+n_{\text {th }} \sigma_{F}^{N}$, где $Q_{F}^{N}-$ среднее по камере значение шумового сигнала ${ }^{3}$ за тайм-фрейм (период

\footnotetext{
3 Знак усреднения опущен: здесь и далее если не указано явно, что речь идет о пиксельной карте (массиве), и явно не указаны пиксельные индексы, имеется ввиду среднее значение массива.
}

интегрирования сигнала) $\Delta t, \sigma_{F}^{N}-$ среднеквадратичное отклонение шумового сигнала в камере, величина $n_{t h}$ определяет уровень достоверности сигнала и, следуя работам [17,51], в настоящих расчетах ее значение принято $n_{\mathrm{th}}=4$. Следует отметить, что величина $Q_{F}^{N}$ может быть легко оценена на аппаратном уровне и в режиме реального времени, поскольку фактически представляет собой отношение суммарного сигнала в камере за тайм-фрейм к количеству пикселей. При этом измерение шума в случае необходимости можно производить постоянно со скоростью $\sim 1 \mathrm{~Hz}$. Величина $\sigma_{F}^{N}$ может быть оценена по формуле $\sigma_{F}^{N}=\sqrt{Q_{F}^{N}}$, что является следствием естественного предположения о пуассоновском характере распределения шума, которое может быть обосновано как теоретически, так и посредством численных расчетов (см., например, разд. 5 настоящей работы), а также проверено непосредственно в ходе эксперимента (при прямом измерении фона ночного неба камерой телескопа).

2) Выбор (определение) порогового значения количества ярких (т.е. удовлетворяющих критерию $\left.\left(Q_{F}^{p / g}+Q_{F}^{N}\right)_{i k} \geq Q_{F}^{\text {th }}\right)$ пикселей, $N_{F}^{\text {th }}$, при достижении или превышении которого осуществляется запись. Это значение может быть весьма различным для разных телескопов, разных режимов работы конкретного телескопа и специфики решаемой задачи $[34,52,53]$. В рамках настоящих расчетов были исследованы характеристики телескопа для пороговых значений $N_{F}^{\text {th }}=3-5$ (подробнее о причинах такого выбора см. разд. 5).

3) Выбор (определение) взаимного расположения ярких пикселей: например, это могут быть любые пиксели камеры, любые пиксели в пределах одного кластера, соседние пиксели - тесные соседи (когда каждый яркий пиксел имеет в окрестности не менее двух ярких пикселей) или просто соседи (когда достаточно соседства хотя бы с одним ярким пикселем), которые образуют связную („остров“) или несвязную область. В настоящем моделировании было выбрано условие простого соседства ярких пикселей (подробнее см. разд. 4.2), а область засветки могла быть несвязной (например, две пары ярких пикселей).

С одной стороны, условия формирования триггера должны быть достаточно мягкими для того, чтобы позволить регистировать наиболее слабые события (из всех, которые вообще доступны наблюдению данным телескопом). С другой стороны, условия формирования триггера должны быть достаточно жесткими, чтобы процедура регистрации была достаточно надежной, а скорость ложного счета (т.е. скорость случайного формирования триггера под влиянием фонового излучения) достаточно низкой. Выбранные условия позволяют полностью удовлетворить этим требованиям (подробнее см. разд. 5).

Изображения, отобранные для записи после выдачи триггера, подвергаются дальнейшей обработке 
с помощью специального кода для анализа данных (см. разд. 4.2).

\section{2. Алгоритм очистки изображений}

Как правило, в черенковских гамма-телескопах детальный анализ записанных изображений осуществляется вне режима реального времени, с помощью чисто программных, а не аппаратных средств. В этом отношении код, использованный в настоящей работе для анализа модельных изображений, фактически является действующим прототипом рабочего варианта кода для детального анализа реальных наблюдательных данных.

Несмотря на то что в настоящее время моделирование обработки данных еще не реализовано в полном объеме и пакет TAIGA Soft позволяет смоделировать очистку изображения черенковской вспышки ШАЛ от шума, вызванного фоном ночного неба, но еще не позволяет полностью вычислить параметры Хилласа и определить параметры первичной частицы, он уже позволяет оценить ряд важных параметров изображений черенковских вспышек ШАЛ: размер ШАЛ [параметр Size, ph.e.] количество фотоэлектронов, сгенерированных излучением ШАЛ в камере, количество пикселей, засвеченных излучением ШАЛ, и т.п., а также таких важных характеристик телескопа как вероятность регистрации полезного события при заданных условиях и пороговая энергия наблюдений.

В настоящих расчетах применен простой алгоритм очистки изображений, состоящий всего из трех шагов:

1) Формирование массива, описывающего изображение черенковской вспышки ШАЛ, зашумленное фоном ночного неба, построенное камерой телескопа за таймфрейм $\Delta t$, за вычетом среднего значения шумового сигнала, т. е. согласно следующей формуле:

$$
\left(q_{F}^{p / g}\right)_{i k}=\left(Q_{F}^{p / g}\right)_{i k}+\left(Q_{F}^{N}\right)_{i k}-Q_{F}^{N} .
$$

Вычитание среднего значения шумового сигнала $Q_{F}^{N}$ из исходной пиксельной карты позволяет в дальнейшем более корректно связать суммарный сигнал в засвеченных пикселях с энергией первичной частицы (это особенно важно для энергий, близких к пороговым).

2) Обнуление тех элементов массива $\left(q_{F}^{S}\right)_{i k}$, значение которых меньше, чем $T=n_{T} \sigma_{F}^{N}$, где величина $n_{T}$ определяет уровень достоверности того, что пиксель засвечен черенковским излучением ШАЛ, а не фоновым излучением ночного неба, т.е. сигнал от этого пикселя является полезным. В настоящей работе было принято значение $n_{T}=4$.

3) Обнуление тех элементов массива $\left(q_{F}^{S}\right)_{i k}$, которые соответствуют изолированным пикселям, т.е. тем, для которых все соседние пиксели имеют величины $\left(q_{F}^{S}\right)_{i k}$, равные нулю после выполнения второго шага).

Следует отметить, что более распространенная версия алгоритма очистки (image cleaning, см. например, $[44,45,54,55])$ отличается от использованной в на- стоящих расчетах. Распространенный вариант предполагает введение не одного порога учета засвеченных пикселей, а двух: $T_{u}, T_{l}$ (индексы $l$ и $u$ означают lower и upper соответственно) [ph.e./pixel], так что $T_{l}<T_{u}$. Причем учет пикселей с засветкой, превышающей $T_{l}$, происходит только в случае, если они являются соседями ярких пикселей с засветкой, превышающей $T_{u}$. При этом распространенный вариант процедуры очистки не предполагает исключения изолированных пикселей. Таким образом, оригинальный алгоритм очистки (при условии $\left.T=T_{U}\right)$ предъявляет несколько более жесткие требования к пикселям, которые могут составить финальное (обработанное) изображение черенковской вспышки ШАЛ по сравнению с распространенным вариантом, поскольку не допускает использования менее достоверных $\left(T_{L} \leq\left(q_{F}^{S}\right)_{i k}<T_{U}\right)$ и очень ярких $\left(T_{U} \leq\left(q_{F}^{S}\right)_{i k}\right)$, но изолированных пикселей. Таким образом, при одинаковом исходном изображении в финальном изображении, сформированном с помощью оригинального алгоритма, будет меньше пикселей, чем в изображении, сформированном с помощью распространенной версии алгоритма, но при этом они с большей достоверностью будут соответствовать полезному сигналу (т.е. будут засвечены именно черенковским излучением ШАЛ).

Также следует отметить, что анализирующий код пакета TAIGA Soft уже сейчас при необходимости позволяет реализовать более сложные процедуры в составе оригинального алгоритма очистки, такие как усреднение с заданным окном, восстановление заданной окрестности (т.е. любого заданного размера) вокруг ярких пикселей (безотносительно яркости самих восстанавливаемых пикселей) и т.п. Эти возможности были доступны уже в пакете ALEGRO Soft для моделирования большого черенковского телескопа ALEGRO [13,36], однако в настоящих расчетах эти возможности, также как и введение двух пороговых значений для учета пикселей в обработанном изображении, не были использованы по следующим соображениям: используемый алгоритм должен быть максимально простым и надежным, чтобы обеспечить пусть грубые, но надежные оценки ключевых характеристик телескопа с модернизированной камерой. В принципе использование каждой из упомянутых дополнительных процедур может позволить смягчить „требования“ к характеристикам первичной частицы, например, дополнительно понизить значение пороговой энергии. Но при этом их применение может привести к снижению достоверности уровня сигнала в пикселях, составляющих обработанное изображение и соответственно, в конечном итоге, вызвать значительные ошибки в оценке параметров первичной частицы. Таким образом, чтобы избежать значительного искажения финального изображения, корректность использования каждой из этих процедур должна быть исследована дополнительно. Например, если ввести в оригинальный алгоритм дополнительный порог (например, $3 \sigma_{F}^{N}$ ) для учета не очень ярких пикселей (таких, что $3 \sigma_{F}^{N} \leq\left(q_{F}^{S}\right)_{i k}<4 \sigma_{F}^{N}$ ), то оценки показывают следующее: при использовании 
фильтра основного диапазона вероятность того, что учтенный в финальном изображении не очень яркий пиксель, примыкающий к „острову“ из 3-4 ярких пикселей, будет ложным, составляет около 5\%, а при использовании фильтра ZWB3 - около 15\%. Если дополнительный порог составляет $2 \sigma_{F}^{N}$, то вероятность появления ложного пикселя, примыкающего к „острову“ из 3-4 ярких пикселей, составит уже около 40\% при использовании фильтра основного диапазона и около 60\% при использовании фильтра ZWB3. Такой вероятный негативный эффект становится особенно важным для изображений событий от частиц вблизи пороговых энергий, когда финальное изображение заведомо содержит небольшое (3-6) количество пикселей и поэтому каждый ложный пиксель значительно искажает его.

В общем случае величины $n_{t h}$ и $n_{T}$, определяющие пороговые значения срабатывания триггера и учета пикселей в финальном изображении, не обязаны быть равными. Но в рамках настоящих расчетов это так $\left(n_{\mathrm{th}}=n_{T}=4\right)$, и вместе с условием исключения изолированных ярких пикселей это позволяет использовать алгоритм очистки изображений также и для определения доли событий, вызывающих срабатывание триггера на запись. Это возможно, поскольку при наличии всего трех ярких пикселей они могут войти в финальное изображение, только если являются соседями (что удовлетворяет условию выработки триггера).

Финальные изображения $q_{F}^{S}$ уже могут быть использованы для вычисления параметров Хилласа [25], описывающих морфологию изображений черенковских вспышек ШАЛ, и определения параметров первичной частицы (ее типа, энергии и направления прихода).

\section{3. Величины, характеризующие сигнал}

Анализирующий код пакета TAIGA Soft позволяет рассчитать ряд статистических характеристик сигнала, которые, в свою очередь, позволяют оценить возможности телескопа по наблюдению космических частиц высоких энергий. Среди этих характеристик были использованы следующие:

1) Средний сигнал $Q_{F}^{S}$ [ph.e./pixel] в исходном изображении черенковской вспышки ШАЛ - количество фотоэлектронов на пиксел за тайм-фрейм $\Delta t=15 \mathrm{~ns}$, усредненное по засвеченным пикселям в области углов $\theta \leq \theta_{E A S} \simeq 1.3^{0}$ вокруг наиболее яркого пикселя, ${ }^{4}$ и затем усредненное по реализациям ШАЛ.

2) Среднеквадратичное отклонение $\sigma_{F}^{S}$ среднего сигнала, вычисленное по реализациям ШАЛ.

3) Вероятность регистрации события $P_{F}^{S}$, оцененная как доля событий, вызвавших срабатывание триггера

\footnotetext{
${ }^{4}$ Величина $2 \theta_{E A S} \simeq 2.6^{0}-$ типичный угловой размер ШАЛ [51]. Суммарная засветка области в фокальной плоскости, ограниченной угловым размером $\theta_{E A S}$ вокруг наиболее яркого пикселя, составляет более $80 \%$ от общей засветки камеры черенковским излучением ШАЛ во всем исследованном диапазоне энергий.
}

на запись, в общем количестве проанализированных событий.

4) Средний размер ШАЛ (параметр $\operatorname{Size}_{F}^{S}$ ) - среднее по реализациям ШАЛ общее количество фотоэлектронов, сгенерированных в камере телескопа черенковским излучением ШАЛ, и его среднеквадратичное отклонение.

5) Среднее по по реализациям ШАЛ общее количество пикселей $N_{F}^{S}$, засвеченных черенковским излучением ШАЛ, и его среднеквадратичное отклонение.

6) Среднее по по реализациям ШАЛ количество ярких $\left(\left(q_{F}^{S}\right)_{i k} \geq T\right)$ пикселей $N_{F}^{S, b r}$ (т. е. количество пикселей в финальном изображении), и его среднеквадратичное отклонение. ${ }^{5}$

\section{5. Результаты моделирования и обсуждение}

\section{1. Основные результаты}

Моделирование отдельных ШАЛ дает ключевые исходные данные для проведения дальнейшего многочастичного моделирования методом Монте-Карло транспорта излучения в оптическом тракте телескопа в каждом конкретном смоделированном событии. Это позволяет осуществить визуализацию отдельных событий и построить плотности распределений различных параметров регистрируемых событий.

Визуализация регистрируемых событий - важный промежуточный результат моделирования наблюдений на черенковских гамма-телескопах. Она позволяет на примере обработки отдельных событий качественно оценить эффективность и адекватность работы алгоритма очистки изображения, т. е. выделения полезного сигнала от черенковского излучения ШАЛ на фоне шумового сигнала ночного неба. Также визуализация позволяет оценить разброс величины сигнала в отдельных пикселях и качественно оценить морфологию изображений черенковских вспышек для различных значений энергий и типов первичных частиц.

На рис. 3,4 представлены изображения в фокальной плоскости телескопа двух отдельных (разных) событий - черенковских вспышек ШАЛ, вызванных вертикально падающими гамма-квантами с энергией $0.3162 \mathrm{TeV}$ (близкой к нижней границе аналитической оценки пороговой энергии) на расстоянии $120 \mathrm{~m}$ от оси ШАЛ. Рис. 3 иллюстрирует процесс регистрации события от гамма-кванта с использованием фильтра основного диапазона $(260-600 \mathrm{~nm})$, а рис. 4 - с использованием фильтра ZWB3 (270-400 nm). Эти изображения демонстрируют как возможности регистрации шумового

\footnotetext{
${ }^{5}$ Строго говоря, все введенные индексы необходимы для однозначного соответствия используемых величин и рассматриваемых ситуаций (тип ШАЛ, используемый фильтр), но при представлении данных (в частности, на осях графиков) для сокращения записи эти индексы (а также и символ усреднения) будут опущены там, где это не может привести к неоднозначному толкованию.
} 


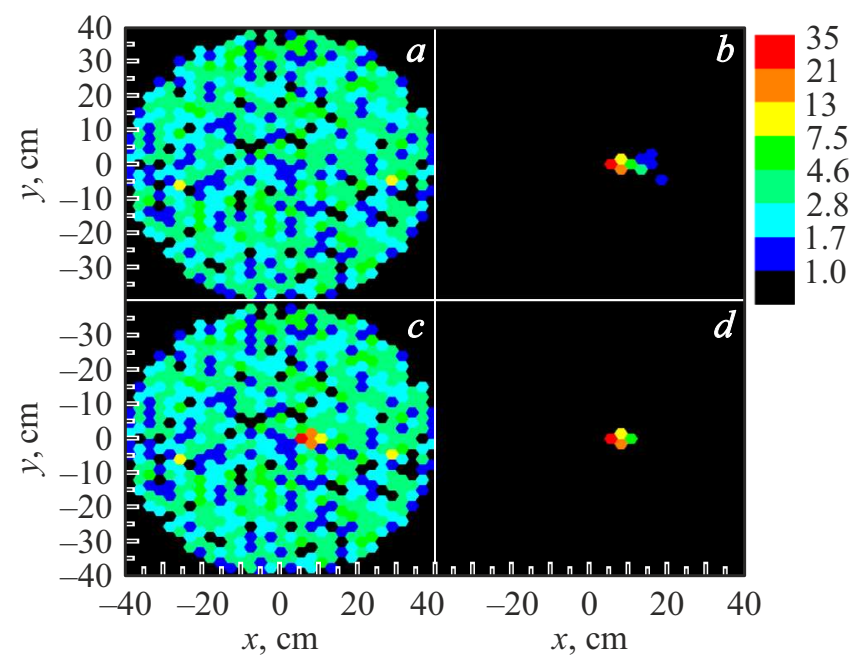

Рис. 3. Пример изображений, формируемых черенковской вспышкой ШАЛ и фоном ночного неба в камере телескопа при использовании фильтра основного диапазона $(260-600 \mathrm{~nm})$ : $a$ - шумовой сигнал, $\left(Q_{\text {opt }}^{N}\right)_{i k}$, сгенерированный в камере оптическим фоном ночного неба; $b$ - исходный полезный сигнал, $\left(Q_{\mathrm{opt}}^{g}\right)_{i k}$, сгенерированный черенковским излучением на расстоянии $120 \mathrm{~m}$ от оси ШАЛ, вызванного вертикально падающим гамма-квантом с энергией $0.3162 \mathrm{TeV} ; c$ - суммарное изображение $a$ и $b-$ модель реального сигнала $\left(Q_{\mathrm{opt}}^{g}+Q_{\mathrm{opt}}^{N}\right)_{i k}$; $d$ - изображение $c$ после очистки от шумового сигнала (обработки с помощью разработанного алгоритма очистки, см. пояснения в тексте), $\left(q_{\mathrm{opt}}^{g}\right)_{i k}$.

сигнала $\left(Q_{F}^{N}\right)_{i k}$, сгенерированного в камере телескопа оптическим фоном ночного неба (рис. 3, $a, 4, a$ ), и полезного сигнала $\left(Q_{F}^{g}\right)_{i k}$, сгенерированного черенковским излучением ШАЛ (рис. $3, b, 4, b$ ), по отдельности, так и возможности регистрации суммарного сигнала $\left(\left(Q_{F}^{g}\right)_{i k}+\left(Q_{F}^{N}\right)_{i k}\right)$, т. е. модели реального сигнала, генерируемого в камере телескопа (рис. 3, c, 4,c), а также показывают результат работы алгоритма очистки изображения $\left(q_{F}^{g}\right)_{i k}$, т. е. выделения полезного сигнала от черенковского излучения ШАЛ на фоне шумового сигнала ночного неба (рис. $3, d, 4, d$ ). Сравнение $3, b, 4, b$ и $3, d, 4, d$ позволяет качественно оценить работу алгоритма очистки изображения как вполне адекватную, о чем говорят следующие обстоятельства: а) все пиксели, засвеченные только фоном ночного неба (т. е. содержащие только шумовой сигнал), отсеяны (исключены) в результате работы алгоритма; б) в финальном изображении нет ложных пикселей, т.е. пикселей, засвеченных фоном ночного неба, но по уровню засветки и расположению имитирующих пиксели, засвеченные черенковской вспышкой, в такой степени, что алгоритм очистки идентифицирует их как пиксели, содержащие полезный сигнал; в) все наиболее яркие пиксели исходного изображения, засвеченные черенковским излучением ШАЛ (т.е. содержащие полезный сигнал), оставлены (учтены) в обработанном (финальном) изображении; г) отсеянные (исключенные) пиксели, содержащие полезный сигнал, являются относительно слабыми по уровню сигнала они сравнимы с шумом и в среднем в 6-9 раз слабее ярких пикселей. Причем ситуация, когда более яркий пиксел исходного изображения был исключен в финальном изображении, а более тусклый в то же время учтен, отсутствует.

В изображении 3, полученном с использованием фильтра основного диапазона $(260-600 \mathrm{~nm})$, общее количество фотоэлектронов, сгенерированных черенковским излучением в камере, составляет Size $=70$ ph.е., из них на яркие пиксели (т.е. те, которые дают вклад в финальное изображение) приходится 63 ph.e., а на тусклые (т. е. отсеянные алгоритмом очистки) - 7 ph.е. (т. е. $10 \%$ от общей величины исходного сигнала). Причем оценка суммарного сигнала в финальном изображении с оставляет 63.6 ph.e. (дробное число появляется вследствие вычитания среднего значения в формуле (7)), т.е. соответствует суммарной яркости исходных пикселей с точностью лучше $1 \%$, что является очень хорошим результатом. Максимальное искажение уровня сигнала в отдельном пикселе (т.е. отличие величины сигнала в пикселе исходного изображения от величины сигнала в соответствующем пикселе финального изображения) составляет менее $15 \%$.

В изображении 4, полученном с использованием фильтра ZWB3 (270-400 nm), общее количество фотоэлектронов, сгенерированных черенковским излучением в камере, составляет $\mathrm{Size}=23$ ph.e., из них на яркие

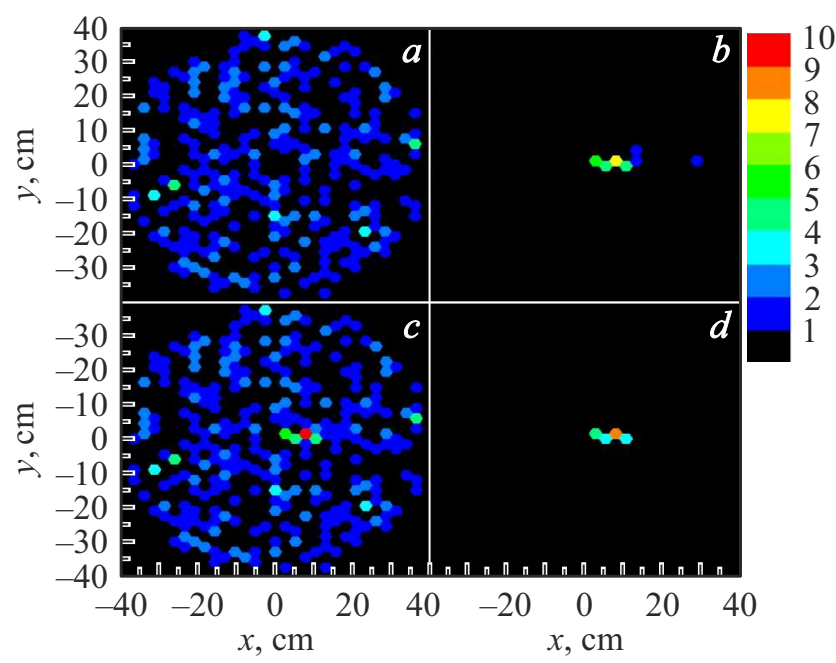

Рис. 4. Пример изображений, формируемых черенковской вспышкой ШАЛ и фоном ночного неба в камере телескопа при использовании фильтра типа ZWB3 $(270-400 \mathrm{~nm}): a$ - шумовой сигнал, $\left(Q_{\mathrm{ZWB}}^{N}\right)_{i k}$, сгенерированный в камере оптическим фоном ночного неба; $b-$ исходный полезный сигнал, $\left(Q_{\mathrm{ZWB}}^{g}\right)_{i k}$, сгенерированный черенковским излучением на расстоянии 120 m от оси ШАЛ, вызванного вертикально падающим гаммаквантом с энергией $0.3162 \mathrm{TeV} ; c-$ суммарное изображение $a$ и $b-$ модель реального сигнала $\left(Q_{\mathrm{ZWB}}^{g}+Q_{\mathrm{ZWB}}^{N}\right)_{i k} ; d-$ изображение $c$ после очистки от шумового сигнала (обработки с помощью разработанного алгоритма очистки, см. пояснения в тексте), $\left(q_{\mathrm{ZWB}}^{g}\right)_{i k}$. 
пиксели приходится 20 ph.e., а на тусклые - 3 ph.e. (т. е. менее $15 \%$ от общей величины исходного сигнала). Оценка суммарного сигнала в финальном изображении составляет 19.82 ph.е., т.е. также соответствует суммарной яркости исходных пикселей с точностью лучше $1 \%$, что так же как и в случае использования фильтра основного диапазона является хорошим результатом. Наибольшее искажение уровня сигнала в отдельном пикселе составляет около $21 \%$.

Несколько худшее согласие обработанных (финальных) изображений с исходными в случае использования фильтра ZWB3 (270-400 nm) естественно, так как относительные флуктуации шума, вызванного фоном ночного неба, больше, чем при использовании фильтра основного диапазона (260-600 nm), а размер ШАЛ, т. е. суммарный сигнал от черенковского излучения ШАЛ в камере, значительно (почти в два раза) меньше.

Важность этих примеров (рис. 3,4) состоит, прежде всего, в том, чтобы показать, что гамма-кванты с энергией $0.3162 \mathrm{TeV}$ способны вызывать срабатывание триггера $\left\{n_{T}=4, N_{F}^{\text {th }}=3\right\}$ на запись. Но, кроме того, они могут вызывать генерацию в модифицированной камере изображений, содержащих 4 (и даже более) ярких пикселей, причем на энергии $0.3162 \mathrm{TeV}$ доля таких событий составляет около $\simeq 10 \%$, а на энергии $0.5 \mathrm{TeV}$ возрастает до $\simeq 40 \%$.

Интересно отметить также следующее обстоятельство: в приведенных на рис. 3,4 событиях сохранено значительное количество важной информация о событии (т.е. об исходном сигнале), что можно оценить как из визуализации, так и из приведенных выше численных параметров этих изображений. В частности, качественно морфология исходных изображения изменена достаточно слабо, что позволяет надеяться на проведение, в дальнейшем, достаточно корректного определения параметров Хилласа. Это, в свою очередь, должно позволить определять параметры первичных частиц с приемлемым уровнем погрешности.

Помимо адекватной работы алгоритма очистки, важным критерием для выбора порогового значения сигнала является следующий: скорость ложного счета (триггирования) должна быть приемлемой для последующей скоростной обработки, т.е. с такой, чтобы электроника могла в режиме реального времени отбраковывать ложные события и не допускать их сохранения в постоянной памяти, чтобы избежать ее переполнения. Ложное срабатывание триггера вызвано фоном ночного неба, под влиянием которого в камере случайным образом складываются подходящие условия: появляются три близких ярких пикселя. И хотя вероятность такого события в каждом конкретном тайм-фрейме весьма мала $\left(\lesssim 10^{-3}\right)$, но из-за малой величины тайм-фрейма $\Delta t=15 \mathrm{~ns}$ общая скорость может оказаться довольно большой. Оценки показывают, что для наиболее мягкого типа триггеpa $\left\{n_{T}=4, N_{F}^{\text {th }}=3\right.$ (neighbors) $\}$ скорость ложного счета при использовании фильтра основного диапазона составит $\simeq 5 \mathrm{~Hz}$ в одном кластере камеры (28 пикселей) и $\simeq 10^{2} \mathrm{~Hz}$ во всей камере. Это практически совпадает со скоростью ложного счета текущей конфигурации камеры TAIGA-IACT $\sim 10^{2} \mathrm{~Hz}[16]$, т. е. является вполне приемлемым значением. При использовании фильтра ZWB3 скорость ложного счета составит $\simeq 1.3 \mathrm{kHz}$ в одном кластере камеры и $\simeq 26 \mathrm{kHz}$ во всей камере. Такая величина скорости ложного счета является неприемлемо высокой величиной ${ }^{6}$ и, следовательно, соответствующий тип триггера $\left\{n_{T}=4, N_{Z W B 3}^{\text {th }}=3\right.$ (neighbors) $\}$ должен быть исключен из списка предполагаемых для использования. Очевидным путем снижения скорости ложного счета является ужесточение условий срабатывания триггера. Насколько эффективно это может быть осуществлено, видно из значений скорости ложного счета всей камеры (560 пикселей), $R_{\text {false }}[\mathrm{Hz}]$, для разных условий срабатывания триггера (табл. 1). Ввиду низкой вероятности реализации таких событий в пределах одного тайм-фрейма, эти значения довольно сложно получить в результате прямого моделирования методом Монте-Калро (можно лишь установить грубые верхние пределы на скорость ложного счета). Но их можно оценить аналитически в предположении пуассоновского распределения шума (именно эти оценки приведены в сводной табл. 1). Величина $\left[Q_{F}^{t h}+1\right]-$ округленное в большую сторону значение порогового сигнала показывает при каком минимальном реальном значении сигнала от пикселя произойдет срабатывание триггера. Скорость ложного счета одного кластера (28 пикселей) примерно в 20 раз меньше, чем скорость ложного счета всей камеры в целом. ${ }^{7}$

Однако следует отметить, что есть и другие способы достаточно быстро на аппаратном уровне отбраковывать ложные изображения. Например, отличительной особенностью истинных изображений черенковского излучения ШАЛ из трех ярких пикселей является то, что на них всегда присутствует очень яркий пиксел (более чем на $7 \sigma$ превосходящий средний уровень шума). Такое дополнительное промежуточное (между первичной и вторичной обработками) условие может быть проверено достаточно быстро и не потребует изменения основных условий срабатывания триггера. Таким образом, с точки зрения приемлемости скорости ложного счета, рассматриваемые типы триггеров представляются вполне удовлетворительными.

Более полное, чем использование оценок, основанных на средних значениях сигналов, представление о возможностях телескопа по первичной регистрации полезного события (выработке триггера на запись) дает

\footnotetext{
6 Текущий вариант проекта допускает возможность того, что „мертвое“ время системы первичной обработки данных может составить до $\simeq 500 \mu \mathrm{s}$, т.е. даже исходя из технических ограничений, общая скорость счета телескопа не должна превышать $\simeq 2 \mathrm{kHz}$, не говоря уже об ограничениях, накладываемых далее процессами хранения и детальной обработки полученных изображений.

7 Текущий проект предусматривает испытание 1-3 модернизированных кластеров на SiPM, поэтому в ходе реализации проекта условия срабатывания триггера могут быть выбраны несколько более мягкими, чем при работе полностью модернизированной камеры.
} 
Таблица 1. Оценка скорости ложного счета, $R_{\text {false, }}$ всей детекторной камеры для различных типов триггера

\begin{tabular}{c|c|c|c|l}
\hline $\begin{array}{c}\text { Тип } \\
\text { фильтра }\end{array}$ & $n_{T}$ & $\begin{array}{c}\text { Порог } \\
{\left[Q_{F}^{\text {th }}+1\right], \text { ph.e. }}\end{array}$ & $\begin{array}{c}\text { Количество пикселей } \\
N_{F}^{\text {th }}(\text { соседи })\end{array}$ & $R_{\text {false, }}, \mathrm{Hz}$ \\
\hline opt & 4 & 10 & 3 & $\simeq 10^{2}$ \\
opt & 4 & 10 & 4 & $\simeq 0.3$ \\
opt & 4 & 10 & 5 & $\simeq 10^{-3}$ \\
opt & 5 & 11 & 3 & $\simeq 1.2$ \\
opt & 5 & 11 & 4 & $\simeq 10^{-3}$ \\
opt & 5 & 11 & 5 & $\simeq 10^{-6}$ \\
ZWB3 & 4 & 4 & 3 & $\simeq 2.6 \cdot 10^{4}$ \\
ZWB3 & 4 & 4 & 4 & $\simeq 6 \cdot 10^{2}$ \\
ZWB3 & 4 & 4 & 5 & $\simeq 14$ \\
ZWB3 & 5 & 5 & 3 & $\simeq 34$ \\
ZWB3 & 5 & 5 & 4 & $\simeq 0.1$ \\
ZWB3 & 5 & 5 & 5 & $\simeq 2 \cdot 10^{-4}$
\end{tabular}

вероятность регистрации в зависимости от энергии первичной частицы. Результаты расчетов этой величины при наблюдении на расстоянии $120 \mathrm{~m}$ от оси ШАЛ для различных типов частиц, используемых фильтров и порогового количества пикселей, представлены на рис. 5 (ромбы соответствуют использованию фильтра основного диапазона, квадраты - ZWB3). Там же представлены зависимости вероятности регистрации от энергии первичной частицы, аппроксимированные с помощью следующей формулы:

$$
P \simeq\left(1+\left(E / E_{0}\right)^{-\alpha}\right)^{-1}
$$

где $E_{0}$ и $\alpha-$ параметры аппроксимации, значения которых приведены в табл. 2. Как уже обсуждалось выше, одной из важных характеристик черенковских телескопов является пороговая энергия регистрации. Обычно она определяется как энергия, при которой дифференциальная скорость счета, $d R_{\gamma} / d E\left[\mathrm{~Hz} \cdot \mathrm{TeV}^{-1}\right]$, от источника, находящегося в зените, со степенным спектром, пропорциональным $E^{-2.5}\left[\mathrm{~cm}^{-2} \cdot \mathrm{s}^{-1} \cdot \mathrm{TeV}^{-1}\right]$ (соответствующим спектру Крабовидной туманности, [56] и ссылки там же), достигает максимума [52,53]. Дифференциальная скорость счета определяется произведением потока гамма-излучения от источника на эффективную площадь детектирования

$$
\frac{d R_{\gamma}}{d E} \sim E^{-2.5} S_{\text {eff }}(E) .
$$

Эффективная площадь детектирования может быть определена по формуле

$$
S_{\mathrm{eff}}(E) \simeq 2 \pi \int_{0}^{\infty} P(E, r) r d r .
$$

Поскольку в настоящей работе были произведены расчеты только для значения $r=120 \mathrm{~m}$, прямое использование выражения (9) для определения пороговой

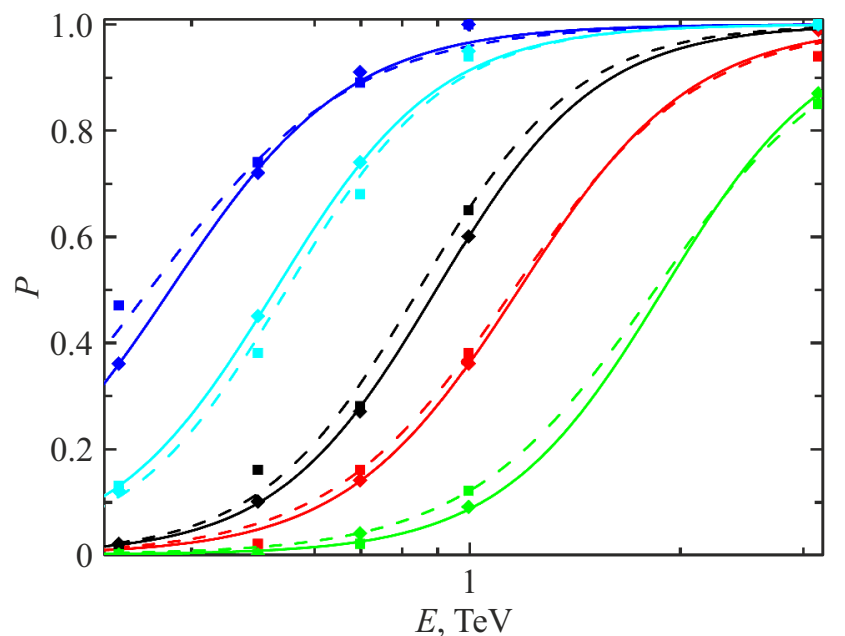

Рис. 5. Вероятность первичной регистрации (выработки триггера на запись) событий на расстоянии $120 \mathrm{~m}$ от оси соответствующих ШАЛ, вызванных вертикально падающими энергичными космическими частицами. Результаты, полученные методом Монте-Карло при использовании фильтра диапазона $(260-600 \mathrm{~nm})$, показаны ромбами, а соответствующие аппроксимации (см. пояснения в тексте) - сплошными кривыми. Результаты, полученные методом Монте-Карло при использовании фильтра типа ZWB3 (270-400 nm), показаны квадратами, а соответствующие аппроксимации (см. пояснения в тексте) - штриховыми кривыми. Зависимость вероятности регистрации событий, вызванных гамма-квантами, $P_{F}^{g}$ от энергии первичных частиц $E$ при использовании условия $N^{\text {th }}=3$ показана синим цветом, $N^{\text {th }}=4-$ голубым, $N^{\text {th }}=5-$ черным (в online версии). Зависимость вероятности регистрации событий, вызванных протонами, $P_{F}^{p}$ от энергии первичных частиц $E$ при использовании условия $N^{\text {th }}=3$ показана красным цветом, $N^{\text {th }}=5-$ зеленым (в online версии).

энергии здесь невозможно. Поэтому пороговая энергия моделируемого телескопа оценена как энергия, на которой достигает максимума выражение $E^{-2.5} P(E, r){ }^{8}$ Значения пороговой энергии, $E_{\mathrm{th}}$, определенные таким образом, также приведены в табл. 2.

Важными параметрами при принятии решения о возможности детального количественного анализа изображения черенковской вспышки ШАЛ на основе параметров Хилласа являются размер ШАЛ (параметр Size) и количество пикселей, учтенных в финальном изображении. Пороговые значения этих параметров могут значительно различаться для разных телескопов. Так, например, детальный анализ изображений при работе

\footnotetext{
8 Для энергий, близких к пороговой, это эквивалентно использованию приближенного выражения для эффективной площади $S_{\text {eff }}(E) \simeq 2 \pi r P(E, r) \Delta r$ в формуле (9), где $r=120 \mathrm{~m}$, а $\Delta r \sim 100 \mathrm{~m}-$ типичная ширина распределения $r P(r)$ (что можно увидеть на примере зависимостей $P(r)$, приведенных в [34]). Следует отметить, что поскольку $r=120 \mathrm{~m}$ близко к некоторому среднему значению прицельного параметра регистрируемых ШАЛ от первичных частиц с энергиями близкими к пороговой, то такое приближение дает вполне адекватную оценку площади детектирования для соответствующих энергий.
} 
Таблица 2. Параметры аппроксимации формулы (8) для вероятности регистрации и приближенная величина пороговой энергии

\begin{tabular}{c|c|c|c|c|c}
\hline $\begin{array}{c}\text { Тип } \\
\text { частицы }\end{array}$ & $\begin{array}{c}\text { Тип } \\
\text { фильтра }\end{array}$ & $\begin{array}{c}\text { Количество пикселей } \\
N^{\text {th }}\end{array}$ & $\begin{array}{c}E_{0}, \\
\mathrm{TeV}\end{array}$ & $\alpha$ & $\begin{array}{c}E_{\text {th }} \\
\mathrm{TeV}\end{array}$ \\
\hline$\gamma$ & opt & 3 & 0.38 & 3.4 & 0.28 \\
$\gamma$ & opt & 4 & 0.53 & 3.7 & 0.44 \\
$\gamma$ & opt & 5 & 0.90 & 3.8 & 0.76 \\
$\gamma$ & ZWB3 & 3 & 0.35 & 3.0 & 0.20 \\
$\gamma$ & ZWB3 & 4 & 0.55 & 3.8 & 0.46 \\
$\gamma$ & ZWB3 & 5 & 0.85 & 3.85 & 0.72 \\
$\mathrm{p}$ & opt & 3 & 1.18 & 3.5 & 0.91 \\
$\mathrm{p}$ & opt & 5 & 1.90 & 3.7 & 1.56 \\
$\mathrm{p}$ & ZWB3 & 3 & 1.16 & 3.3 & 0.82 \\
$\mathrm{p}$ & ZWB3 & 5 & 1.85 & 3.25 & 1.28
\end{tabular}

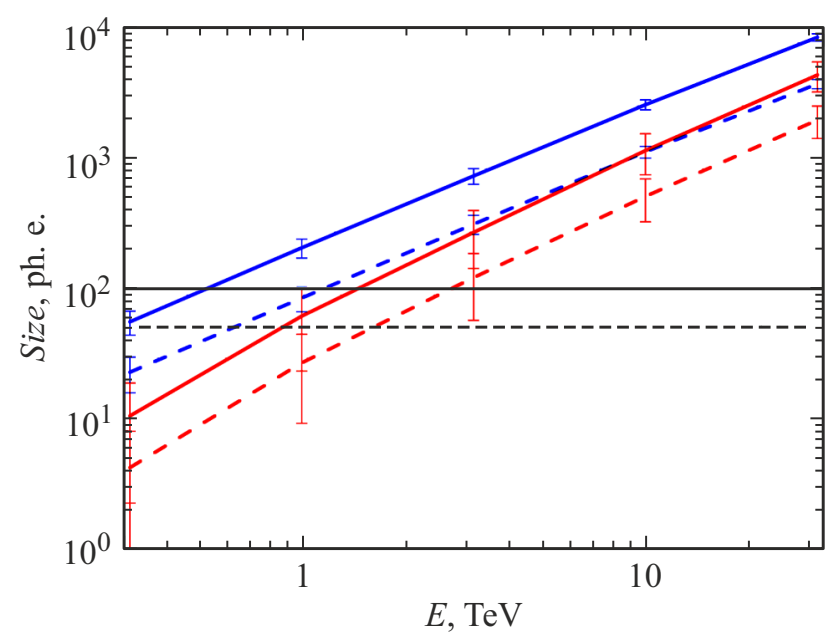

Рис. 6. Общее количество фотоэлектронов, сгенерированных в камере черенковскими фотонами (параметр $\operatorname{Size}_{F}^{S}$ ) на расстоянии $120 \mathrm{~m}$ от оси ШАЛ, вызванных вертикально падающими гамма-квантами (синие кривые) и протонами (красные кривые (в online версии)). Зависимости $\operatorname{Size}_{F}^{S}$, полученные при использовании фильтра основного диапазона $(260-600 \mathrm{~nm})$, показаны сплошными цветными кривыми, при использовании фильтра типа ZWB3 (270-400 nm) - штриховыми цветными кривыми. Пороговые значения $\mathrm{Size}^{\mathrm{Th}}$ показаны черным цветом: уровень $10^{2}$ ph.e. - сплошная кривая, уровень 50 ph.е. - штриховая кривая.

телескопа TAIGA-IACT с камерой на фотоумножителях начинается при превышении параметром Size порогового значения $\mathrm{Size}_{\mathrm{th}}=100$ ph.e. [16], при работе телескопа H.E.S.S. - (40-45) ph.e. (см., например, [45,54]), а при работе телескопа HEGRA, имевшего примерно те же параметры, что и TAIGA-IACT, - 40 ph.e. [52,57]. Результаты расчетов параметра Size для различных типов первичных частиц и используемых типов фильтров представлены на рис. 6. Также на этом рисунке отмечены возможные пороговые значения параметра Size: 50 и 100 ph.e. Величина энергии первичной частицы
$E_{\text {Size, }}$ при которой среднее значение параметра Size достигает пороговых значений при различных режимах наблюдения приведена в табл. 3.

Относительное среднеквадратичное отклонение параметра Size по серии событий от гамма-квантов составляет $21-17 \%$ в интервале энергий $0.3162-1 \mathrm{TeV}$ при наблюдениях с использованием фильтра основного диапазона и $31-22 \%$ в том же интервале энергий при наблюдениях с использованием фильтра ZWB3. Таким образом, распределение параметра Size - достаточно узкое, и его среднее значение примерно соответствует медианному значению, т.е. вероятность превышения среднего значения составляет около $\sim 50 \%$. Это означает, что значения энергии $E_{\mathrm{Size}}$, приведенные в табл. 3, являются надежными оценками пороговой энергии для проведения детального анализа изображений черенковских вспышек ШАЛ при заданном пороговом значении параметра Size $_{\text {th. }}$ Следует отметить, что конкретный выбор порогового значения параметра Size $_{\text {th }}$ зависит от требований, которые предъявляются к качеству окончательных результатов, т.е. следует ориентироваться на приемлемый уровень ошибок измерения энергии первичной частицы и величину углового разрешения, которые могут довольно сильно отличаться в зависимости от конкретного телескопа и специфики решаемой задачи. Расчеты показывают, что для одних и тех же событий параметр Size оказывается примерно в два раза меньше при использовании фильтра ZWB3 (270-400 nm), чем при использовании фильтра основного диапазона (260-600 nm). Таким образом, установка универсального порогового значения Size $\mathrm{th}_{\mathrm{th}}$ ведет к значительному ухудшению пороговой энергии $E_{\text {Size }}$ (при которой возможен детальный анализ изображения) при переходе от режима регистрации с использованием фильтра основного диапазона к регистрации с использованием фильтра типа ZWB3. При этом сам по себе параметр Size является ключевым для определения энергии первичной частицы. В то же время основная часть параметров Хилласа [25] (имеющих геометрическую природу) зависит не от абсолютного размера ШАЛ (параметра Size), а от количества засвеченных пикселей, использованных для анализа. Результаты расчетов общего числа пик-

Таблица 3. Энергия первичной частицы, необходимая для достижения порогового значения параметра Size

\begin{tabular}{c|c|c|l}
\hline $\begin{array}{c}\text { Тип } \\
\text { частицы }\end{array}$ & $\begin{array}{c}\text { Тип } \\
\text { фильтра }\end{array}$ & $\begin{array}{c}\text { Пороговое значение } \\
\text { Size }_{\text {th }}\end{array}$ & $\begin{array}{l}E_{\text {Size }}, \\
\text { TeV }\end{array}$ \\
\hline$\gamma$ & opt & 100 & 0.54 \\
$\gamma$ & opt & 50 & 0.3 \\
$\gamma$ & ZWB3 & 100 & 1.17 \\
$\gamma$ & ZWB3 & 50 & 0.64 \\
p & opt & 100 & 1.5 \\
p & opt & 50 & 0.88 \\
p & ZWB3 & 100 & 2.76 \\
p & ZWB3 & 50 & 1.63
\end{tabular}




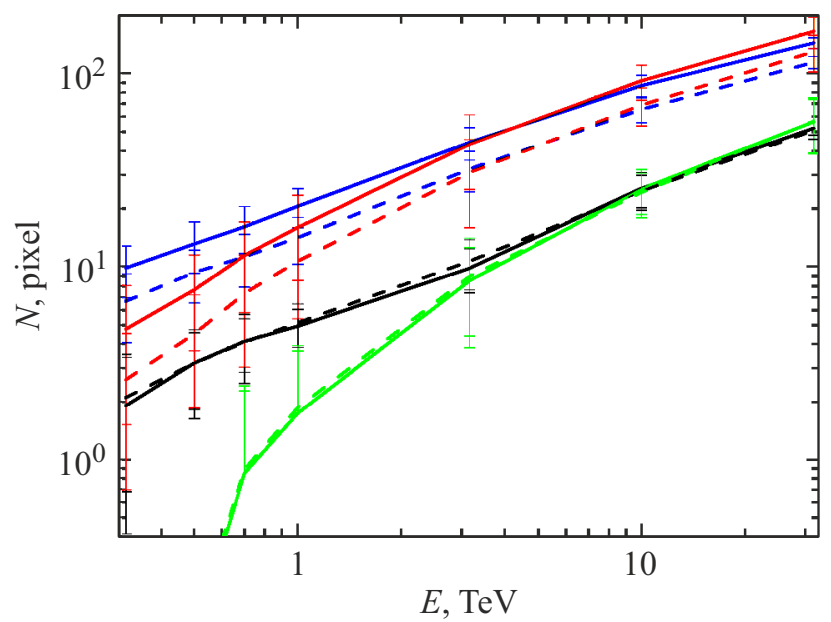

Рис. 7. Общее количество засвеченных пикселей (синие кривые соответствуют событиям, вызванным гамма-квантами, красные - протонами (в online версии) и количество неизолированных ярких пикселей (сигнал в которых превышает пороговое значение $Q_{F}^{\text {th }}$, черные кривые соответствуют событиям, вызванным гамма-квантами, зеленые - протонами (в online версии)) при регистрации событий на расстоянии $120 \mathrm{~m}$ от оси ШАЛ. Сплошные кривые соответствуют расчетам с учетом использования фильтра основного диапазона $(260-600 \mathrm{~nm})$, штриховые кривые - фильтра ZWB3 (270-400 nm).

селей, засвеченных черенковским излучением ШАЛ, и числа ярких пикселей, которые могут быть учтены в финальном изображении и использованы для анализа параметров Хилласа, приведены на рис 7. Из этого рисунка видно, что зависимости количества ярких пикселей от энергии при использовании фильтра основного диапазона и при использовании фильтра типа ZWB3 практически совпадают, ${ }^{9}$ несмотря на то, что значения параметра Size для одной и той же энергии отличаются примерно в два раза. Таким образом, если уровень точности определения энергии первичной частицы (который определяется флуктуациями параметра Size) около 30\% является приемлемым, то при использовании фильтра ZWB3 целесообразно устанавливать пороговое значение параметра Size на уровне Size $_{\text {th }}=50$ ph.e. Установка порогового значения Size $_{\mathrm{th}}=100$ ph.е. при использовании фильтра типа ZWB3 приведет к неоправданному увеличению порога регистрации.

Пороговое значение по количеству пикселей в финальном изображении, $N_{\mathrm{Th}}$, при достижении которого можно производить анализ параметров Хилласа, также может быть различным для разных телескопов (например, при работе H.E.S.S., как правило, используется $\left.N_{\mathrm{Th}}=4[45,58]\right)$. Как уже упоминалось выше (см. разд. 4.2), при наиболее распространенном варианте алгоритма очистки изображения (см. например, [44,54]),

\footnotetext{
9 Это вполне ожидаемо уже из сравнения поведения вероятностей регистрации $P_{\text {opt }}^{S}(E)$ и $P_{Z W B}^{S}(E)$ (рис. 6), которые также отличаются весьма слабо.
}

количество таких пикселей может значительно отличаться от количества пикселей, которые вызвали срабатываение триггера. Но поскольку конкретный вариант алгоритма очистки, представленного в настоящей работе (см. разд. 4.2), использует для учета пикселей то же пороговое значение, которое используется при выработке триггера, то в рамках настоящих расчетов именно пиксели, вызвавшие срабатывание триггера, и составят финальное изображение, т. е. $N_{\mathrm{Th}}=N^{\text {th }}$. Поэтому пороговая энергия для достижения заданного количества пикселей финального изображения, при которой возможно проведение анализа параметров Хилласа, будет в данном случае совпадать с пороговой энергией первичной регистрации (срабатывания триггера) $E_{\mathrm{th}}$, приведенной в табл. 2. Следует учитывать, что наличие всего трех ярких пикселей в финальном изображении явно недостаточно для проведения эффективного анализа параметров Хилласа (поскольку полученные значения будет практически невозможно применить для гамма-адронной сепарации и определения параметров первичной частицы с приемлемой точностью). Тем не менее, изучение характеристик телескопа для триггера $N^{\text {th }}=3$ отнюдь не бессмысленно по следующим причинам: 1) дальнейшее применение распространенного алгоритма очистки $[45,55]$ с двумя порогами учета пикселей позволяет обрабатывать такие изображения и количество пикселей в финальном изображении будет больше трех (хотя вызвать срабатывание триггера могут, например, только три из них) и количество информации будет достаточным для проведения детального анализа; ${ }^{10}$ 2) нельзя исключать возможность регистрации гамма-излучения по превышению сигнала над шумом (как это планировалось в телескопах первого поколения [59-61], при этом гамма-адронная сепарация не требуется) для очень ярких источников и при условии, что не требуется высокое угловое разрешение и точность определения энергии первичной частицы. Но даже использование алгоритма очистки, описанного в разд. 4.2, для $N_{\mathrm{Th}}=N^{\text {th }}=4$ (в соответствии с аналогичным условием работы телескопа H.E.S.S. [45]) приводит к вполне приемлемому значению пороговой энергии $\simeq 0.45 \mathrm{TeV}$ (если игнорировать ограничения со стороны параметра Size). Финальные изображения, содержащие пять и более пикселей, можно назвать „высококачественными“, поскольку такое количество информации практически гарантирует эффективность анализа параметров Хилласа и, соответственно, корректное определение параметров первичной частицы.

Таким образом, проведенные исследования показывают следующее:

1) Пороговая энергия первичной регистрации космических гамма-квантов (выработки триггера на запись данных) от источников с малыми зенитными углами находится в интервале $0.28-0.76 \mathrm{TeV}$ в зависимости от используемого типа триггера и используемого ти-

\footnotetext{
${ }^{10}$ Изучение возможностей применения двухпорогового алгоритма очистки будет выполнено в ходе дальнейших исследований.
} 
па фильтра. При этом максимальная (из приемлемых значений) скорость ложного счета достигается для мягкого типа триггера $\left\{n_{T}=4, N^{\text {th }}=4\right.$ (neighbors) $\}$ при использовании фильтра ZWB3 (что дает минимальный сигнал для выдачи триггера $\left[Q_{\mathrm{ZWB}}^{\text {th }}+1\right]=4$ ph.e. $/$ pixel $)$ и составляет около $0.6 \mathrm{kHz}$ (что является вполне приемлемым значением). Следует также отметить, что скорость ложного счета экспоненциально быстро убывает при ужесточении любого из условий триггирования.

2) Пороговая энергия вторичной регистрации гаммаквантов (эффективного анализа параметров Хилласа и корректного определения параметров первичной частицы) от источников с малыми зенитными углами находится в интервале $0.3-0.76 \mathrm{TeV}$ в зависимости от используемого сочетания условий $\mathrm{Size}_{\mathrm{th}}, N_{\mathrm{Th}}$, используемого типа фильтра и применяемого алгоритма очистки, причем условие Size $\mathrm{ZWB}_{\mathrm{th}}^{\mathrm{th}}=100$ ph.e. может быть сочтено нецелесообразно жестким, так как оно приводит к неоправданному росту пороговой энергии регистрации.

При использовании фильтра основного диапазона, сочетание наиболее жестких (из рассмотренных) пороговых условий первичной и вторичной обработки $\left\{n_{T}=4\right.$, $N^{\text {th }}=N_{\text {Th }}=5$ (neighbors), Size $_{\text {th }}=100$ ph.e. $\}$ приводит к значению пороговой энергии $0.76 \mathrm{TeV}$. При использовании фильтра типа ZWB3, сочетание наиболее жестких пороговых условий $\left\{n_{T}=4, N^{\text {th }}=N_{\mathrm{Th}}=5\right.$ (neighbors), Size $_{\text {th }}=50$ ph.e. $\}$ приводит к значению пороговой энергии $0.72 \mathrm{TeV}$. Оба значения примерно в два раза меньше пороговой энергии текущей конфигурации телескопа TAIGA-IACT с камерой на вакуумных фотоумножителях XР1911, которая составляет около $\sim 1.5 \mathrm{TeV}$ [16].

\section{2. Сравнение с результатами полуаналитических оценок}

Как уже упоминалось выше, моделирование различных режимов работы телескопа и предварительные оценки его параметров уже выполнялись в работе [17] с использованием полуаналитической модели формирования, распространения (см. также [29]) и регистрации черенковского излучения ШАЛ. В этой модели использовались величины, позволяющие наглядно (хотя и приближенно) оценить возможности регистрации черенковского излучения ШАЛ камерой черенковского телескопа: средний полезный сигнал $Q_{F}^{g / p}$, сгенерированный в камере черенковским излучением ШАЛ, и средний шумовой сигнал $Q_{F}^{N}$, а также его среднеквадратичное отклонение по пикселям камеры $\sigma_{F}^{N}$ (вычисленное в предположении пуассоновского распределения фоновых фотонов, попадающих на входное окно пикселя). Для сравнения с результатами полуаналитической модели [17], проверки ее применимости и дальнейшей корректировки указанные величины были оценены и в рамках настоящих расчетов. Результаты расчетов этих величин, а также среднеквадратичного отклонения полезного сигнала по серии событий $\sigma_{F}^{g / p}$ (которое было невозможно оценить

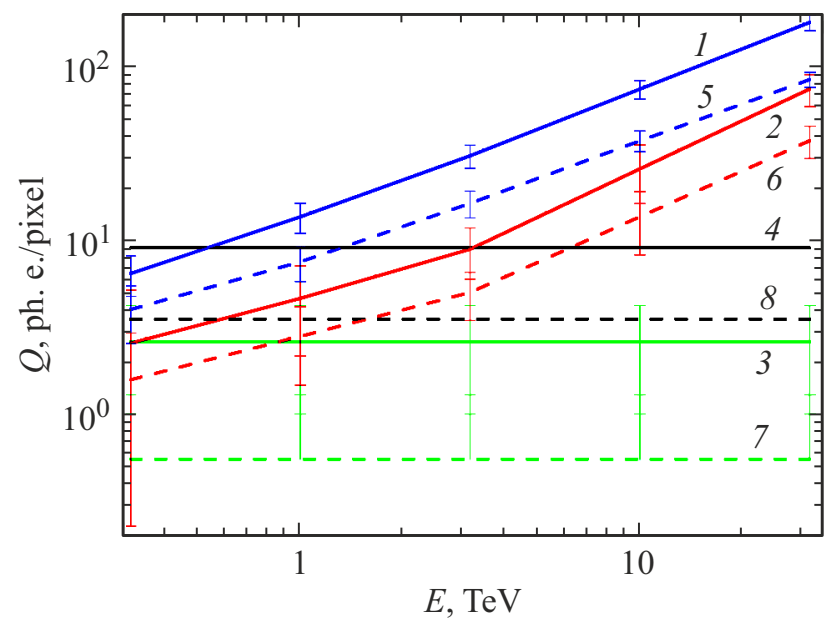

Рис. 8. Зависимости среднего полезного и шумового сигналов от энергии первичной частицы: 1 - полезный сигнал, $Q_{\mathrm{opt}}^{g} \pm \sigma_{\mathrm{opt}}^{g}$, от событий, вызванных гамма-квантами, при регистрации с использованием фильтра основного диапазона $(260-600 \mathrm{~nm}) ; 2$ - полезный сигнал, $Q_{\mathrm{opt}}^{p} \pm \sigma_{\mathrm{opt}}^{p}$, от событий, вызванных протонами, при регистрации с использованием фильтра основного диапазона; 3 - шумовой сигнал, $Q_{\mathrm{opt}}^{N} \pm \sigma_{\mathrm{opt}}^{N}$, вызванный фоном ночного неба, при регистрации с использованием фильтра основного диапазона; 4 - пороговое значение $Q_{\mathrm{opt}}^{\text {th }}$ для записи сигнала при регистрации с использованием фильтра основного диапазона; 5 - полезный сигнал, $Q_{\mathrm{ZWB}}^{g} \pm \sigma_{\mathrm{ZWB}}^{g}$, от событий, вызванных гамма-квантами, при регистрации с использованием фильтра ZWB3 $(270-400 \mathrm{~nm})$; 6 - полезный сигнал, $Q_{\mathrm{ZWB}}^{p} \pm \sigma_{\mathrm{ZWB}}^{p}$, от событий, вызванных протонами, при регистрации с использованием фильтра ZWB3; 7 - шумовой сигнал, $Q_{\mathrm{ZWB}}^{N} \pm \sigma_{\mathrm{ZWB}}^{N}$, вызванный фоном ночного неба, при регистрации с использованием фильтра ZWB3; 8 пороговое значение $Q_{Z \mathrm{th}}^{\text {th }}$ для записи сигнала при регистрации с использованием фильтра ZWB3 (см. пояснения в тексте).

в рамках модели [17]), представлены на рис. 8. Ожидаемый уровень среднего полезного сигнала при регистрации событий от первичных гамма-квантов с использованием фильтра основного диапазона $(260-600 \mathrm{~nm})$ монотонно нарастает (примерно в соответствии со степенной зависимостью) от $(6.4 \pm 1.7)$ ph.e./pixel при энергии $316.2 \mathrm{GeV}$ до $(178 \pm 19)$ ph.e./pixel при $31.62 \mathrm{TeV}$ (рис. 8). Ожидаемый уровень среднего полезного сигнала от первичных протонов при использовании фильтра основного диапазона $(260-600 \mathrm{~nm})$ монотонно нарастает от $(2.6 \pm 2.6)$ ph.e./pixel при энергии $316.2 \mathrm{GeV}$ до $(73.7 \pm 15.4)$ ph.e./pixel при $31.62 \mathrm{TeV}$ (рис. 8). Величина шумового сигнала, вызванного в отдельном пикселе фотонами оптического фона ночного неба при использовании фильтра основного диапазона $(260-600 \mathrm{~nm})$ за время регистрации $15 \mathrm{~ns}$ составляет $(2.6 \pm 1.6)$ ph.e./pixel (рис. 8). Ожидаемый уровень среднего полезного сигнала при регистрации событий от первичных гамма-квантов с использованием фильтра типа ZWB3 (270-400 nm) монотонно нарастает (также примерно в соответствии со степен- 
ной зависимостью) от $(4 \pm 1.5)$ ph.e./pixel при энергии $316.2 \mathrm{GeV}$ до $(83.4 \pm 8.3)$ ph.e./pixel при $31.62 \mathrm{TeV}$ (рис. 8). Ожидаемый уровень среднего полезного сигнала от первичных протонов при использовании фильтра типа ZWB3 $(270-400 \mathrm{~nm})$ монотонно нарастает от $(1.6 \pm 1.4)$ ph.e./pixel при энергии $316.2 \mathrm{GeV}$ до $(37.2 \pm 7.8)$ ph.e./pixel при $31.62 \mathrm{TeV}$ (рис. 8). Величина шумового сигнала, вызванного в отдельном пикселе фотонами оптического фона ночного неба при использовании фильтра типа ZWB3 $(270-400 \mathrm{~nm})$ за время регистрации $15 \mathrm{~ns}$ составляет $(0.55 \pm 0.74)$ ph.e./pixel (pис. 8). При использовании среднего сигнала $Q_{F}^{S}$ для оценки пороговой энергии наблюдений телескопа, естественным приближенным условием, имитирующем реальный критерий для выработки триггера $\left\{n_{T}=4, N^{\text {th }}=3\right\}$ на запись данных в постоянную память, является условие превышения средним сигналом $Q_{F}^{g / p}$ порогового значения $Q_{F}^{\text {th }}$ [17]. Для обоснования применимости указанной формулировки приближенного условия выработки триггера в [17] использовался тот факт, что превышение средним значением порогового значения гарантирует, что среднее значение по трем наиболее ярким пикселям также превышено. Также при этом были использованы следующие предположения: а) среднее значение по трем наиболее ярким пикселям не будет сильно (не более чем в 2-4 раза) отличаться от среднего по всем засвеченным пикселям; б) при этом превышение порогового значения произойдет в каждом из трех наиболее ярких пикселей; в) типичная морфология изображений приведет к тому, что наиболее яркие пиксели будут соседями. При работе с фильтром основного диапазона $(260-600 \mathrm{~nm})$ значение порогового сигнала составляет $Q_{\mathrm{opt}}^{\mathrm{th}} \simeq 9$ ph.e./pixel (рис. 8 ), а при работе с фильтром типа ZWB3 $(270-400 \mathrm{~nm})-Q_{\mathrm{ZWB}}^{\mathrm{th}} \simeq 3.5$ ph.e./pixel (рис. 8). Такие значения порогового сигнала приводят к следующим оценкам пороговой энергии: при работе с фильтром основного диапазона $(260-600 \mathrm{~nm})$ значение пороговой энергии регистрации гамма-излучения составляет $\sim 0.54 \mathrm{TeV}$ (рис. 8 ), а при работе с фильтром типа ZWB3 $(270-400 \mathrm{~nm})-0.3 \mathrm{TeV}$ (рис. 8). Нетривиальный эффект - возможность понижения пороговой энергии наблюдений при использовании УФ-фильтра [17] — связан с тем, что УФ-фильтр типа ZWB3 подавляет шумовой сигнал по сравнению с фильтром основного диапазона $(260-600 \mathrm{~nm})$ сильнее, чем полезный сигнал. Расчеты, проведенные в настоящей работе, показывают, что при использовании фильтра типа ZWB3 пороговое значение сигнала уменьшается примерно в 2.6 раза, в то время как полезный сигнал от гамма-квантов в окрестности значения пороговой энергии подавляется примерно в 1.7 раза. Полученные значения пороговой энергии наблюдений находятся в очень хорошем согласии с результатами [17] (пороговая энергия для основного диапазона была оценена на уровне $0.6 \mathrm{TeV}$, a при использовании фильтра типа ZWB3 $-0.3 \mathrm{TeV})$. При этом следует отметить, что в оценке величины среднего

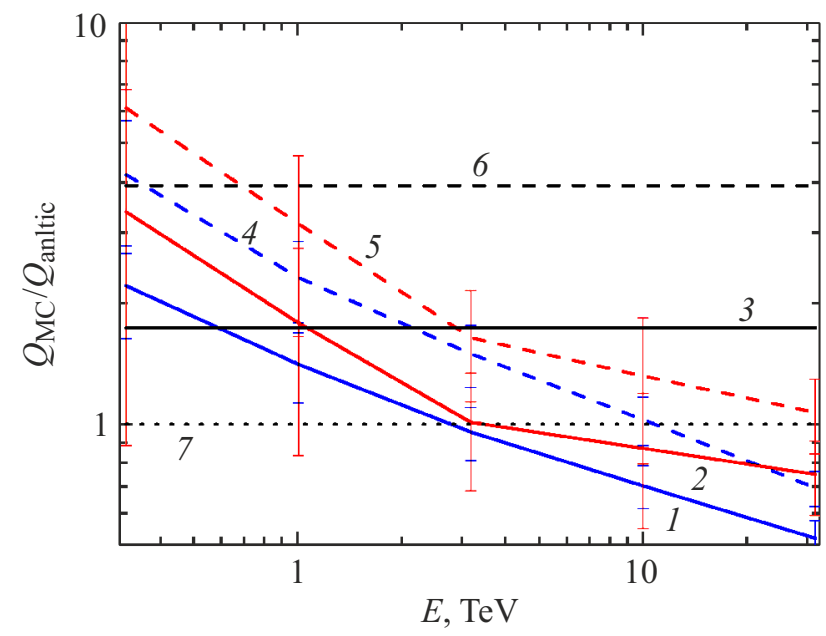

Рис. 9. Отношения величин сигналов, вычисленных в рамках настоящей работы методом Монте-Карло (нижний индекс „МС“), к соответствующим величинам, оцененным полуаналитически в рамках работы [17] (нижний индекс ,anltc“): 1 $\left(Q_{\mathrm{opt}}^{g} \pm \sigma_{\mathrm{opt}}^{g}\right)_{\mathrm{MC}} /\left(Q_{\mathrm{opt}}^{g}\right)_{\mathrm{anltc}} ; 2-\left(Q_{\mathrm{opt}}^{p} \pm \sigma_{\mathrm{opt}}^{p}\right)_{\mathrm{MC}} /\left(Q_{\mathrm{opt}}^{p}\right)_{\mathrm{anltc}} ;$ $3-\left(Q_{\mathrm{opt}}^{\text {th }}\right)_{\mathrm{MC}} /\left(Q_{\mathrm{opt}}^{\text {th }}\right)_{\text {anltc }} ; 4-\left(Q_{\mathrm{ZWB}}^{g} \pm \sigma_{\mathrm{ZWB}}^{g}\right)_{\mathrm{MC}} /\left(Q_{\mathrm{ZWB}}^{g}\right)_{\text {anltc }}$; $5-\left(Q_{\mathrm{ZWB}}^{p} \pm \sigma_{\mathrm{ZWB}}^{p}\right)_{\mathrm{MC}} /\left(Q_{\mathrm{ZWB}}^{p}\right)_{\mathrm{anltc}} ; 6-\left(Q_{\mathrm{ZWB}}^{\mathrm{th}}\right)_{\mathrm{MC}} /\left(Q_{\mathrm{ZWB}}^{\mathrm{th}}\right)_{\mathrm{anltc}}$; 7 - единичный уровень.

сигнала наблюдаются значительные отличия. Для иллюстации этих отличий на рис. 9 представлены отношения средних сигналов $Q_{F}^{S}$, рассчитаных в рамках настоящего исследования методом Монте-Карло (индекс „МС“), к соответствующим величинам, оцененным в работе [17] (индекс „anltc“). ${ }^{11}$ При изменении энергии первичной частицы от 0.3162 до $31.62 \mathrm{TeV}$ значения среднего полезного сигнала от гамма-квантов, полученные в текущих расчетах, отличаются от значений [17] в $2.2-0.5$ раза для фильтра основного диапазона $(260-600 \mathrm{~nm})$ и в 4.2-0.7 раза для фильтра ZWB3 (рис. 9). Те же отличия для сигнала от протонов составляют $3.4-0.75$ и 6.1-1.07 соответственно (рис. 9). Отличия текущих результатов от результатов [17] обусловлены тем, что, как уже упоминалось выше, модели формирования, распространения и регистрации черенковского излучения ШАЛ , использованные для расчетов [17], изначально были основаны на большом количестве приближений (физических, математических и методических). Так, для описания продольного профиля (т.е. зависимости количества от глубины атмосферы) электронов ШАЛ от гамма-квантов использовалось приближение Грей-

\footnotetext{
${ }^{11}$ Следует отметить, что, поскольку при проведении расчетов [17] использовались приближенные полуаналитические модели формирования и регистрации черенковского излучения ШАЛ [17], они позволяли оценить только уровень среднего сигнала $Q_{F}^{N}$, и не позволяли определить его среднеквадратичное отклонение $\sigma_{F}^{N}$ по серии событий. В этом отношении показатели уровня сигнала, рассчитанные в настоящей работе, также как и результаты, полученные в [17], дают общее, но при этом более полное представление о возможностях регистрации черенковского излучения ШАЛ телескопом TAIGA-IACT с модернизированной камерой на SiPM.
} 
зена [62], а от протонов космических лучей - приближение Грейзена-Ильиной-Линсли [63]. Рождение и распространение черенковского излучения описывалось с помощью редуцированного уравнения переноса [29] (уравнения переноса, проинтегрированного по ряду параметров), что, в частности, не позволяло описать угловое распределение черенковских фотонов ШАЛ. Моделирование оптического тракта телескопа [17] также осуществлялось приближенными методами: взаимодействие пучка фотонов с узлами телескопа учитывалось посредством умножения количества фотонов в пучке на коэффициенты передачи узлов, т.е. вероятностный характер взаимодействия не мог быть принят во внимание. Кроме того, ограниченность (неполнота) описания модели [17] привела к необходимости использования методических приближений, таких как использование приближенной формулировки условия на выдачу триггера или применение оценок типа $\overline{f(x)} \simeq f(\bar{x})$ (где $f-$ регулярная функция случайной величины $x$ ), которые в общем случае не верны, но используются, поскольку более точное вычисление величины $\overline{f(x)}$ требует знания плотности распределения $x$ (или хотя бы достаточного количества ее моментов), чего используемая модель обеспечить не может. Среди конкретных причин расхождений (которые являются непосредственными следствиями грубости использованных приближений) можно выделить следующие. Из-за невозможности рассчитать угловое распределение черенковского излучения ШАЛ, количество засвеченных пикселов $N_{\text {anltc }}$ в модели [17] считалось постоянным и определялось типичным угловым размером ШАЛ $\left(\sim 2.6^{0}\right)$, что при угловом размере пикселя $0.36^{0}$ приводит к значению $N_{\text {anltc }} \simeq 47$. При многочастичном моделировании методом Монте-Карло (настоящий расчет) факт засветки каждого конкретного пиксела определяется непосредственно в процессе симуляции транспорта фотонов в оптическом тракте телескопа вплоть до их взаимодействия с фотоэлементами камеры.

Результаты расчетов общего количества пикселей, засвеченных черенковским излучением ШАЛ, представлены на рис. 7. С учетом того обстоятельства, что $Q_{\mathrm{MC}} / Q_{\text {anltc }} \simeq\left(\right.$ Size $\left._{\mathrm{MC}} / \mathrm{Size}_{\text {anltc }}\right)\left(N_{\text {anltc }} / N_{\mathrm{MC}}\right)$, из представленных данных можно сделать вывод, что отличие количества засвеченных пикселей $N_{\text {MC, }}$ рассчитанного методом Монте-Карло, от $N_{\text {anltc }}$ является основной причиной отличия значения среднего сигнала $Q_{\mathrm{MC}}$ от значения $Q_{\text {anltc }}$ аналитически оцененного в [17]. Следует отметить, что отличие $N_{\mathrm{MC}}$ от $N_{\text {anltc }}$ обусловлено не меньшим угловым размером ШАЛ, чем $2.6^{0}$, a, скорее, тем, что на энергиях, близких к нижней границе наблюдательного диапазона, абсолютное количество фотонов в пучке черенковского излучения ШАЛ становится недостаточным. Общий фактор конверсии телескопом фотонов в фотоэлектроны сигнала составляет $\simeq 0.2$ ph.e./photon при использовании фильтра основного диапазона и $\simeq 0.1$ ph.e./photon при использовании фильтра типа ZWB3. Таким образом, при умень- шении энергии первичной частицы и соответственно, интенсивности черенковской вспышки, краевые пиксели изображения черенковской вспышки (соответствующие краям диаграммы направленности черенковского излучения ШАЛ) перестают засвечиваться раньше, чем центральные пиксели, несмотря на то, что на них все еще падает довольно значительное количество фотонов (5-10 штук).

Помимо использования различных приближений, в работе [17] также отличаются некоторые исходные данные. При формулировке модели [17] не предполагалось использование фильтра основного диапазона (однако диапазон, для которого производились вычисления, был ограничен длинами волн 240-600 nm), поэтому коэффициент пропускания фильтра основного диапазона $F_{\text {opt }}(\lambda)=0.8$ в интервале $(260 \lesssim \lambda \lesssim 600 \mathrm{~nm})$, использование которого предполагается в настоящей работе, также оказывает соответствующее влияние на оцененный уровень среднего сигнала $Q_{\text {opt }}^{S}$. Длительность времени регистрации отдельного события (тайм-фрейм) в [17] составляла $10 \mathrm{~ns}$, а в настоящей работе была увеличена до $15 \mathrm{~ns}$. Это было сделано, чтобы учесть тот факт, что на расстояниях $\gtrsim 150 \mathrm{~m}$ от оси ШАЛ длительность черенковской вспышки может значительно превышать $10 \mathrm{~ns}$ (см., например, [64]), т.е. использование такого окна регистрации может привести к существенному недоучету полезного сигнала, в то время как тайм-фрейм $\Delta t=15 \mathrm{~ns}$ обеспечивает учет не менее $80 \%$ полезного сигнала при всех реальных значениях расстояния до оси ШАЛ.

Средний уровень шумового сигнала, оцененный в работе [17], составлял 2.14 ph.e./pixel при работе в основном диапазоне и 0.37 ph.e./pixel при использовании фильтра типа ZWB3. C учетом отличий в исходных данных (увеличенной с 10 до $15 \mathrm{~ns}$ длительности времени регистрации и использования фильтра основного диапазона $\left.F_{\text {opt }}=0.8\right)$ эти результаты с точностью около $1 \%$ соогласуются с результатами, полученными в настоящей работе. Среднеквадратичное отклонение шумового сигнала более значительно отличается от результатов предварительных расчетов [17]: при использовании фильтра основного диапазона его величина больше примерно в 2 раза, а при использовании фильтра типа ZWB3 примерно в 5.8 раза, чем значения, отраженные в [17]. Такое отличие обусловлено несовершенством модели, использованной в [17]: предполагалось пуассоновское распределение фотонов, падающих на входное окно пикселя, но вероятностный характер взаимодействия излучения с отдельными узлами оптического тракта пикселя (защитным покрытием, фильтрами, конусами Уинстона и фотоэлементами) не учитывался, т. е. это взаимодействие рассматривалось как детерменированный процесс. Это приводило к тому, что взаимодействие фотонов с оптическим трактом пикселя не вносило вклад в дисперсию распределения шумовых фотоэлектронов, и распределение уже не рассматривалось как пуассоновское 
(т. е. $\left.\sigma_{F}^{N} \neq \sqrt{Q_{F}^{N}}\right)$. При моделировании методом МонтеКарло (настоящий расчет) взаимодействие каждого фотона с каждым узлом тракта рассматривается как случайный процесс с соответствующей вероятностью, исход которого разыгрывается для каждого такого взаимодействия отдельно. Именно это приводит к дополнительному вкладу в дисперсию шумового сигнала по сравнению с оценками, полученными в расчетах [17], и фактически восстановлению пуассоновского распределения шумовых фотоэлектронов (в частности, восстанавливается условие $\sigma_{F}^{N}=\sqrt{Q_{F}^{N}}$. Изменение в параметрах шума приводит к соответствующему изменению значений порогового сигнала: при использовании фильтра основного диапазона его величина больше примерно в 1.7 раза, а при использовании фильтра типа ZWB3 примерно в 3.9 раза, чем значения, полученные в работе [17]. Таким образом, оценки величины полезного сигнала от гамма-квантов в диапазоне энергий $0.3-1 \mathrm{TeV}$ и величины порогового сигнала выросли соразмерно по сравнению с расчетами [17]. Этим и объясняется очень хорошее согласие значений пороговой энергии с результатами [17].

Таким образом, результаты расчетов соотношения среднего сигнала и шума методом Монте-Карло подтверждают предварительный вывод [17] о возможности значительного снижения пороговой энергии малоразмерного чренковского телескопа типа TAIGA-IACT за счет использования SiPM вместо вакуумных ФЭУ, что также согласуется с выводами, сделанными по результатам более полного и обоснованного анализа данных (также полученных методом Монте-Карло), проведенного в разд. 5.1. При использовании фильтра основного диапазона $(260-600 \mathrm{~nm})$ это объясняется более высоким (примерно в два раза) значением эффективности детектирования SiPM OnSemi MicroFJ-60035, чем у вакуумных фотоумножиелей ХР1911. При использовании фильтра ZWB3 (270-400n்m) это объясняется более мягкими (но тем не менее весьма эффективными) условиями выдачи триггера $\left(Q_{\mathrm{ZWB}}^{\text {th }}=3.5\right.$ ph.e. $/$ pixel $)$ и проведения детального анализа (Size ${ }^{\mathrm{Th}}=50$ ph.e.), чем установленные для текущей конфигурации TAIGA-IACT $\left(Q^{\text {th }}=10\right.$ ph.e. $/$ pixel при превышении в двух соседних пикселях, $\left.\mathrm{Size}^{\mathrm{Th}}=100 \mathrm{ph} . \mathrm{e}[16]\right)$. Также результаты расчетов соотношения среднего сигнала и шума методом Монте-Карло, выполненных в настоящей работе, подтверждают оценку значений пороговой энергии $(\simeq 0.6 \mathrm{TeV}$ при использовании фильтра основного диапазона и $\simeq 0.3 \mathrm{TeV}$ при использовании фильтра ZWB3), сделанную в работе [17], и с точностью до множителя $(1.5-2)$ согласуются со значениями пороговой энергии первичной регистрации с триггером $\left\{n_{T}=4, N^{\text {th }}=3\right\}$, $\simeq 0.28 \mathrm{TeV}$ при использовании фильтра основного диапазона и $\simeq 0.2 \mathrm{TeV}$ при использовании фильтра ZWB3 (при условии игнорирования ограничений со стороны скорости ложного счета, которые также не учитывались в работе [17])), полученными посредством более полного анализа данных, проведенного в разд. 5.1. В то же время, более обоснованный анализ результатов моделирования методом Монте-Карло, проведенный в разд. 5.1, не подтверждает предварительный вывод [17] о выраженном уменьшении значения пороговой энергии при замене фильтра основного диапазона на фильтр ZWB3 (при сохранении прочих условий триггера $\left\{n_{T}, N^{\text {th }}\right\}$ ).

Следует отметить, что несмотря на значительные недостатки, полуаналитическая модель [17] обладает и некоторыми преимуществами: она наглядна (позволяет проследить влияние изменения исходных данных на все промежуточные величины и оценить его корректность) и позволяет быстро производить оценки среднего полезного сигнала и пороговой энергии телескопа с точностью $\sim 50 \%$, параметров шумового сигнала с точностью около $1 \%$, а также правильно выявлять тенденции в изменениях вычисляемых параметров при изменении исходных данных. Поэтому ввиду затратности и большой длительности многочастичных расчетов методом МонтеКарло, при появлении дополнительных обстоятельств в рассматриваемой задаче может быть целесообразно производить предварительные оценки с помощью полуаналитической модели [17] для принятия решения о необходимости проведения полномасштабного моделирования. Например, осуществление методом МонтеКарло даже самых грубых оценок параметров телескопа при изменении любых исходных условий (типа фотоэлемента (что соответственно ведет к изменению зависимости $\operatorname{PDE}(\lambda)$, иногда вплоть до полной смены диапазона чувствительности), параметров питания фотоэлемента (напряжения смещения), типа фильтра или даже коэффициента отражения покрытия конусов Уинстона) требует около 5-7 дней, тогда как получение соответствующих оценок с использованием модели [17] занимает менее 1 часа. Поэтому результаты более полного и обоснованного анализа, проведенного в разд. 5.1 настоящей работы, будут использованы для корректировки и калибровки модели [17] с целью ее использования в дальнейшем для проведения быстрых предварительных расчетов, потребность в которых может возникнуть при проведении работ по проекту модернизации камеры для TAIGA-IACT, особенно с учетом того, что конкретные типы некоторых фильтров еще не определены.

\section{3. Дополнительные результаты}

Помимо изложенных выше основных результатов выполненного моделирования (исследования различных режимов работы телескопа и определение пороговой энергии наблюдений, соответствующей этим режимам) в ходе расчетов были получены существенные дополнительные результаты. К ним можно отнести следующие: 1) оценка максимальной загрузки фотоэлементов; 2) визуализация событий от частиц с энергиями $>3 \mathrm{TeV}$ (для которых надежность регистрации и корректность детальной обработки несомненны), иллюстрирующая различие в морфологии изображений черенковских вспышек ШАЛ от гамма-квантов и от прото- 


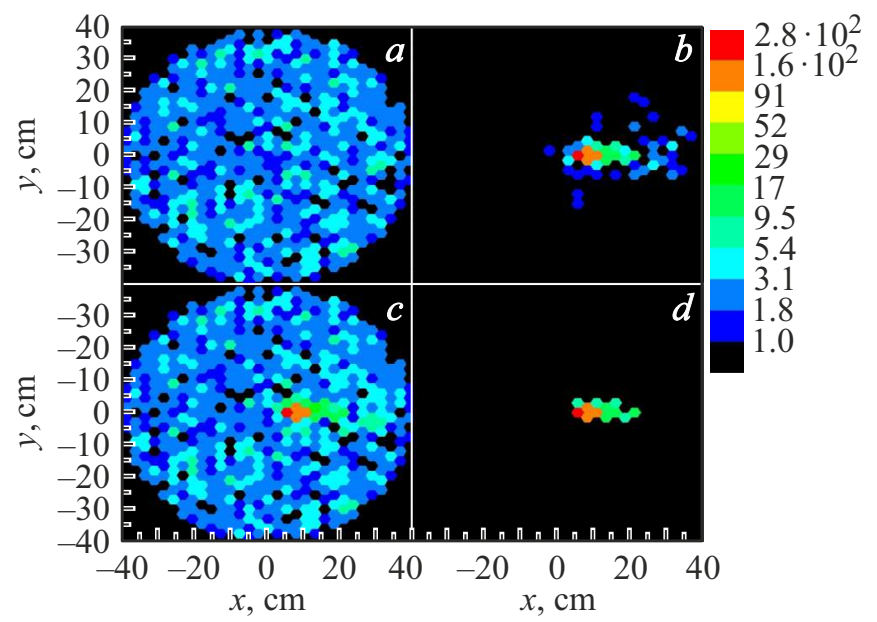

Рис. 10. Пример изображений, формируемых черенковской вспышкой ШАЛ и фоном ночного неба в камере телескопа при использовании фильтра основного диапазона $(260-600 \mathrm{~nm})$ : $a$ - шумовой сигнал, $\left(Q_{\text {opt }}^{N}\right)_{i k}$, сгенерированный в камере оптическим фоном ночного неба; $b$ - исходный полезный сигнал, $\left(Q_{\mathrm{opt}}^{g}\right)_{i k}$, сгенерированный черенковским излучением на расстоянии $120 \mathrm{~m}$ от оси ШАЛ, вызванного вертикально падающим гамма-квантом с энергией $3.162 \mathrm{TeV}$; $c$ - суммарное изображение $a$ и $b-$ модель реального сигнала $\left(Q_{\mathrm{opt}}^{g}+Q_{\mathrm{opt}}^{N}\right)_{i k}$; $d$ - изображение $c$ после очистки от шумового сигнала (обработки с помощью разработанного алгоритма очистки, см. пояснения в тексте), $\left(q_{\mathrm{opt}}^{g}\right)_{i k}$.

нов; 3) оценка возможности повышения эффективности гамма-адронной сепарации путем грубой спектрометрии черенковского излучения ШАЛ посредством использования УФ-фильтра с пропусканием в области длин волн $\lesssim 300 \mathrm{~nm}$.

Максимальная загрузка фотоэлементов при определенной энергии первичной частицы определяется величиной максимального сигнала в отдельном наиболее ярко засвеченном пикселе. В процессе регистрации в основном диапазоне $(260-600 \mathrm{~nm})$ одного события от первичного гамма-кванта с энергией $31.62 \mathrm{TeV}$ эта величина составляет около $(4.4 \pm 0.26) \cdot 10^{3}$ ph.e., что соответствует низкому уровню загрузки фотоэлемента (который будет состоять из 4-х SiPM OnSemi MicroFJ-60035 с общим количеством микроячеек свыше 70000 [65]) и исключает насыщение фотоэлементов и просчет фотонов на энергии $31.62 \mathrm{TeV}$. Экстраполяция результатов говорит о том, что и на энергии $300 \mathrm{TeV}$ (верхней границе рабочего интервала TAIGA-IACT) насыщение маловероятно. Учитывая, что при использовании фильтра типа ZWB3 уровень сигнала, сгенерированного черенковским излучением ШАЛ, примерно в два раза меньше, чем при использовании фильтра основного диапазона (при прочих равных условиях), очевидно, что при использовании фильтра ZWB3 насыщение фотоэлементов практически исключено.

Для полноты демонстрации перспектив работы модернизированной камеры на SiPM была выполнена визуа- лизация модельных изображений черенковских вспышек ШАЛ от первичных космических частиц с энергиями из интервала $>3 \mathrm{TeV}$, в котором надежная регистрация заведомо гарантирована. На рис. 10 представлена визуализация черенковской вспышки ШАЛ от вертикально падающего первичного гамма-кванта с энергией $3.162 \mathrm{TeV}$ при наблюдении с использованием фильтра основного диапазона $(260-600 \mathrm{~nm})$, а на рис. $11-$ визуализация той же самой вспышки при наблюдении с использованием фильтра ZWB3 (260-600 nm). Из сравнения рис. 10 и 11 видно, что различие в исходных изображениях (рис. $10, b, 11, b)$ выражается в изменении конфигурации и количества наиболее слабо засвеченных пикселей (1-4 ph.e.). Причем таких пикселей больше на рис. $10, b$, полученном в основном диапазоне, что неудивительно, поскольку итоговая эффективность детектирования [ph.e./photon] при работе в основном диапазоне выше примерно в два раза, чем при работе в диапазоне фильтра ZWB3. Количество ярких пикселей, составляющих финальное (обработанное) изображение (рис. $10, d, 11, d)$ практически одинаково: 12 пикселей при использовании фильтра основного диапазона и 11 пикселей при использовании фильтра ZWB3. Несмотря на общее уменьшение уровня сигнала при использовании фильтра ZWB3 (что можно увидеть из верхнего значения цветовой шкалы), относительные

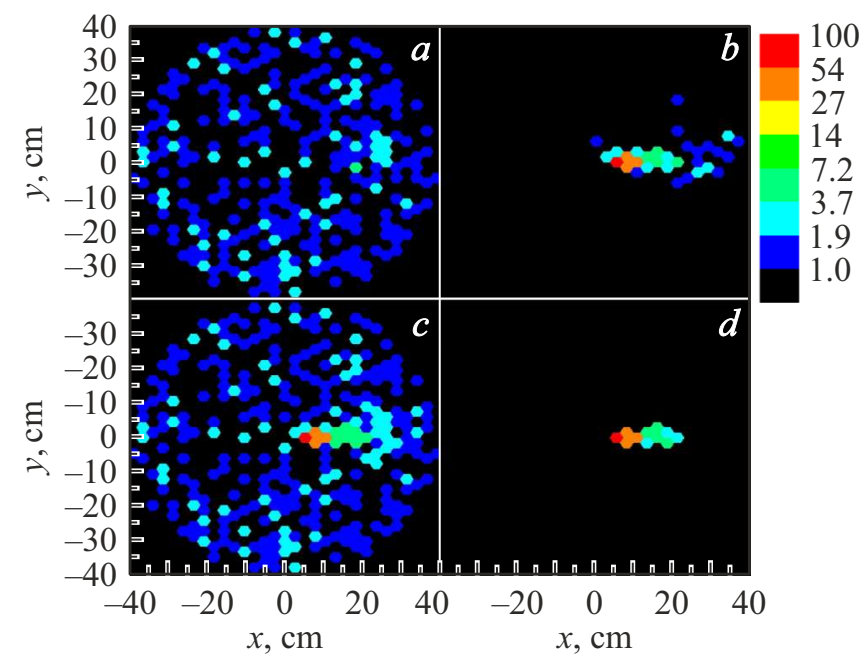

Рис. 11. Пример изображений, формируемых черенковской вспышкой ШАЛ и фоном ночного неба в камере телескопа при использовании фильтра типа ZWB3 $(270-400 \mathrm{~nm}): a-$ шумовой сигнал, $\left(Q_{Z W B}^{N}\right)_{i k}$, сгенерированный в камере оптическим фоном ночного неба (для того же распределения фоновых фотонов, что и на рис. 10$) ; b-$ исходный полезный сигнал, $\left(Q_{\mathrm{ZWB}}^{g}\right)_{i k}$, сгенерированный черенковским излучением на расстоянии $120 \mathrm{~m}$ от оси ШАЛ, вызванного вертикально падающим гамма-квантом с энергией $3.162 \mathrm{TeV}$ (для того же события, что и на рис. 10); $c-$ суммарное изображение $a$ и $b-$ модель реального сигнала $\left(Q_{\mathrm{ZWB}}^{g}+Q_{\mathrm{ZWB}}^{N}\right)_{i k} ; d-$ изображение $c$ после очистки от шумового сигнала (обработки с помощью разработанного алгоритма очистки, см. пояснения в тексте), $\left(q_{\mathrm{ZWB}}^{g}\right)_{i k}$. 
уровни сигнала в пикселях сохраняются (о чем, в свою очередь, свидетельствует преимущественное сохранение распределения цветности пикселей). Также видно, что в значительной степени сохраняется пространственное распределение пикселей (как и в случае с отсеянными пикселями исходного изображения, здесь изменения касаются наиболее слабых из пикселей финального изображения, вес которых в дальнейшей обработке также не является определяющим), т. е. морфология изображения в основном сохранена. Это говорит о том, что определение геометрических параметров Хилласа (размеров фитирующего эллипса, его ориентации и положения центра тяжести) по изображениям рис. $10, b, 11, b$ должно привести практически к одинаковым результатам. При этом следует иметь ввиду, что помимо фактического отличия изображений рис. 10 и 11 (они получены с разными фильтрами), присутствуют еще и случайные отличия, обусловленные вероятностным характером взаимодействия фотонов с узлами оптического тракта телескопа (что естественным образом учитывается при моделировании методом Монте-Карло), т.е. даже два последовательно полученных с использованием одного и того же фильтра изображения одной и той же черенковской вспышки будут несколько отличаться друг от друга.

На рис. 12 представлено модельное изображение, формируемое черенковским излучением ШАЛ от вертикально падающего первичного протона с энергией $10 \mathrm{TeV}$ при наблюдении с использованием фильтра основного диапазона $(260-600 \mathrm{~nm})$. Событие от первич-

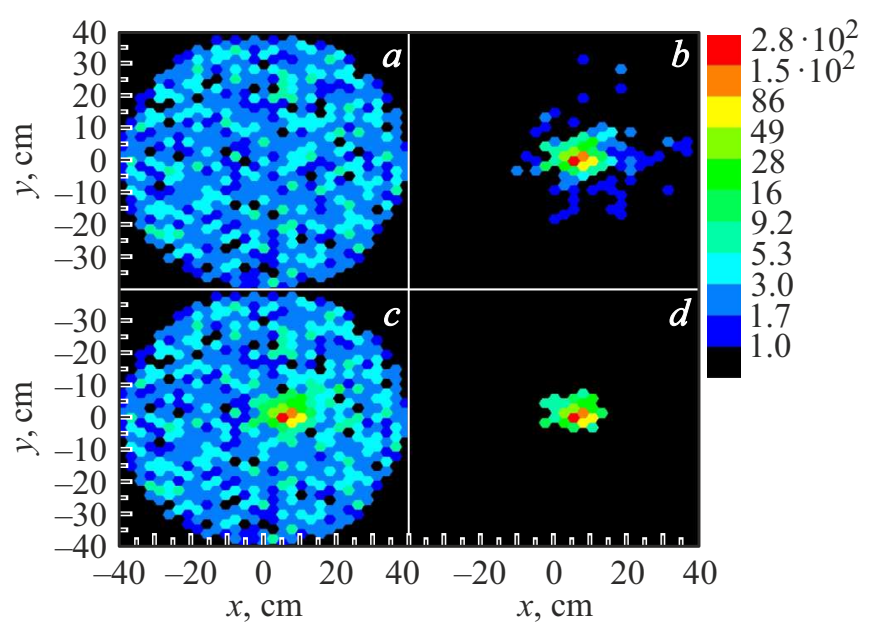

Рис. 12. Пример изображений, формируемых черенковской вспышкой ШАЛ и фоном ночного неба в камере телескопа при использовании фильтра основного диапазона $(260-600 \mathrm{~nm})$ : $a$ - шумовой сигнал, $\left(Q_{\text {opt }}^{N}\right)_{i k}$, сгенерированный в камере оптическим фоном ночного неба; $b$ - исходный полезный сигнал, $\left(Q_{\mathrm{opt}}^{p}\right)_{i k}$, сгенерированный черенковским излучением на расстоянии $120 \mathrm{~m}$ от оси ШАЛ, вызванного вертикально падающим протоном с энергией $10 \mathrm{TeV} ; c-$ суммарное изображение $a$ и $b-$ модель реального сигнала $\left(Q_{\mathrm{opt}}^{p}+Q_{\mathrm{opt}}^{N}\right)_{i k}$; $d$ - изображение $c$ после очистки от шумового сигнала (обработки с помощью разработанного алгоритма очистки, см. пояснения в тексте), $\left(q_{\mathrm{opt}}^{p}\right)_{i k}$. ного протона с таким значением энергии выбрано для визуализации потому, что его энергетические характеристики (параметр Size с учетом статистических ошибок, уровень максимального и среднего сигналов в финальном изображении) достаточно близки к энергетическим характеристикам событий от первичных гамма-квантов с энергией $3.162 \mathrm{TeV}$. Сравнение изображений (рис. 10 и рис. 12) указывает на явную разницу в их морфологии: изображение черенковской вспышки ШАЛ от протона значительно более рассеяно и имеет значительно менее выраженную ориентацию, чем изображение черенковской вспышки ШАЛ от гамма-кванта. Формализация именно этих свойств с помощью параметров Хиллаca [25] и позволяет производить эффективную гаммаадронную сепарацию событий. Например, очевидно, что отношение длин осей фитирующего эллипса изображения события от протона будет значительно отличаться от аналогичного отношения для изображения события от гамма-кванта. Качественная демонстрация этих свойств является важной ввиду их общеизвестности, поскольку подтверждает как корректность выполненного моделирования черенковского излучения ШАЛ с помощью CORSIKA, так и косвенно подтверждает корректность моделирования работы малоразмерного телескопа с камерой на SiPM.

Еще одной важной задачей, которую планируется решить в процессе модернизации камеры телескопа TAIGA-IACT, является повышение эффективности гамма-адронной сепарации посредством измерения параметров черенковского излучения ШАЛ в среднем УФдиапазоне [26-29]. Эту задачу планируется решать с помощью применения соответствующего фильтра MUVдиапазона (рис. 1). Метод гамма-адронной сепарации с использованием УФ-компоненты черенковского излучения ШАЛ основывается на следующем свойстве ШАЛ: при фиксированном значении поверхностной плотности черенковских фотонов ШАЛ (или параметра Size для конкретного телескопа) доля УФ-излучения в диапазоне $\leq 300 \mathrm{~nm}$ по отношению к количеству излучения в основном диапазоне $(260-600 \mathrm{~nm})$ будет больше в случае, если первичной частицей ШАЛ является протон, чем в случае, если первичной частицей ШАЛ является гаммаквант. Это связано с тем, что генерация черенковского излучения ШАЛ от протонов в среднем происходит глубже в атмосфере (т.е. на меньших высотах от поверхности), чем в случае ШАЛ от гамма-квантов. Поэтому черенковские фотоны ШАЛ от протонов проходят меньшую оптическую толщу по поглощению на атмосферном озоне, чем черенковские фотоны ШАЛ от гамма-квантов (подробное изложение этого эффекта см. в $[28,29]$ и ссылки там же). Для практического использования этого эффекта можно использовать УФфильтр с полосой пропускания в диапазоне длин волн $\leq 300 \mathrm{~nm}$ для половины равномерно распределенных пикселей модернизированных SiPM-кластеров камеры, а после регистрации события осуществлять сравнение сигналов от пикселей УФ и полного диапазона. Отно- 
шение этих сигналов будет зависеть от типа первичной частицы. В настоящее время в рамках проекта тип фильтра для диапазона $\leq 300 \mathrm{~nm}$, который можно было бы использовать для решения поставленной задачи, не определен окончательно. Поэтому в настоящей работе для расчетов используется модельный профиль коэффициент передачи гипотетического прямоугольного фильтра с пропускной способностью $80 \%$ в полосе длин волн 260-300 nm (рис. 8), который далее будет обозначен как MUV-фильтр (т.е. „model/middle UVфильтр“б). Ряд исследований показывает, что создание реального фильтра с характеристиками, близкими к требуемым, вполне возможно [66,67]. На основании многочастичного моделирования рассчитано отношение сигналов от черенковских фотонов ШАЛ при использовании фильтра типа MUV (260-300 nm) и при использовании фильтра основного диапазона $(260-600 \mathrm{~nm})$ в зависимости от энергии и типа первичной частицы. При изменении энергии от 0.3162 до $31.62 \mathrm{TeV}$ для сигнала от гамма-квантов это отношение составляет $18-8 \%$, а для сигнала от протонов $11-9 \%$. Сформулированный в [17] предварительный критерий гамма-адронной сепарации энергичных событий по значению доли УФизлучения в черенковском излучении ШАЛ предполагал, что абсолютная разница средних полезных сигналов от гамма-квантов и от протонов при использовании MUV-фильтра должна превышать 1 ph.e. при условии равенства сигналов от гамма-квантов и от протонов в основном диапазоне (260-600 nm) (последнее наступает при условии, что энергия протонов в $3-3.5$ раза больше энергии гамма-квантов). Согласно расчетам [17], такая формулировка критерия приводит к следующему выводу: применение фильтра типа MUV (260-300 nm) может повысить эффективность гамма-адронной сепарации в интервале энергий примерно от 25 до $50 \mathrm{TeV}$. Расчеты, проведенные в настоящей работе показывают, что такая формулировка критерия гамма-адронной сепарации, видимо, является неудовлетворительной. Так, например, полезный средний сигнал от гамма-квантов с энергией $10 \mathrm{TeV}$ в основном диапазоне $(260-600 \mathrm{~nm})$ составляет $(73.5 \pm 9)$ ph.e., а от протонов с энергией $31.62 \mathrm{TeV}-$ $(73.7 \pm 15.4)$ ph.e., т.е. величины этих сигналов практически равны. В то же время полезный средний сигнал от гамма-квантов с энергией $10 \mathrm{TeV}$ при использовании MUV-фильтра составляет (7.0 \pm 1.14$)$ ph.e., a от протонов с энергией $31.62 \mathrm{TeV}-(6.9 \pm 1.6)$ ph.e., т.е. величины этих сигналов также практически совпадают. Таким образом, сравнение средних сигналов в диапазоне $(260-300 \mathrm{~nm})$ не позволяет эффективно отличить гамма-кванты с энергией $10 \mathrm{TeV}$ от протонов c энергией $31.62 \mathrm{TeV}$. Хотя многочастичное моделирование не выполнялось для энергий свыше $31.62 \mathrm{TeV}$ (это весьма затруднительно с точки зрения скорости и объемов данных из-за очень большого количества частиц и ввиду невозможности использовать механизмы упрощения расчетов, такие как объединение фотонов в пучки [bunch]), а для подобных исследований требова- лось бы моделирование ШАЛ от протонов с энергией $\sim 100 \mathrm{TeV}$, тем не менее с учетом флуктуаций значений сигналов сложно ожидать, что ситуация с эффективностью предложенного критерия гамма-адронной сепарации радикально улучшится в диапазоне 25-50 TeV. Это привело к необходимости поиска иного параметра, характеризующего сигнал в диапазоне $(260-300 \mathrm{~nm})$, такого, чтобы его значения разрешались в достаточной степени для различных первичных частиц. В настоящее время подходящим кандидатом на роль такого параметра представляется средний сигнал от трех наиболее ярких пикселей, полученный при использовании MUV-фильтра $(260-300 \mathrm{~nm})$. Средний по трем пикселам полезный сигнал от гамма-квантов с энергией $10 \mathrm{TeV}$ при использовании MUV-фильтра составляет $(20.3 \pm 2.6)$ ph.e., а от протонов с энергией $31.62 \mathrm{TeV}-(29.6 \pm 7.9)$ ph.e., a разность этих величин составляет $(9.3 \pm 8.3)$ ph.e. Таким образом, эти величины статистически разрешены с вероятностью около 70\%, т.е. в большей степени, чем средние сигналы по всем засвеченным пикселам. Это позволяет использовать полезный сигнал, усредненный по трем наиболее ярким пикселам, для повышения эффективности гамма-адронной сепарации. Но, поскольку, как предполагается, фильтр MUV будет использоваться одновременно с фильтром основного диапазона (они будут маскировать пиксели камеры в порядке, максимально близком к шахматному), то использовать именно этот параметр непосредственно не получится, и потребуется дальнейшее уточнение критерия гаммаадронной сепарации с использованием величины сигнала в диапазоне 260-300 nm. Однако полученный результат дает надежду, что окончательная формулировка такого критерия вполне возможна и будет успешно применена на практике. Ожидаемым результатом в этом случае будет явное бимодальное распределение исследуемого параметра (подобное распределению, полученному в [68]), каждая мода которого будет соответствовать определенному типу первичных частиц. Следует отметить, что уровень вероятности разрешения УФ-сигнала от протонов и гамма-квантов $\simeq 70 \%$ сам по себе недостаточен, чтобы послужить основой для самостоятельного метода гаммаадронной сепарации. Но использование превышения сигналом от УФ-излучения ШАЛ порогового значения, как дополнительного критерия отбора, может повысить надежность стандартной процедуры гамма-адроной сепарации посредством анализа параметров Хилласа [25], которая является основной для всех телескопов с многопиксельными камерами и всегда используется при анализе результатов наблюдений черенковских телескопов. Кроме того, дальнейшее уточнение типа регистрируемого параметра черенковского излучения ШАЛ в MUVдиапазоне, должно привести к повышению степени разрешения значений этого параметра для событий от протонов и гамма-квантов, что может дополнительно повысить его роль в общей процедуре гамма-адронной сепарации (или даже сделать его основным параметром для определения типа первичной частицы). 


\section{Заключение}

Проведенные исследования показали, что значение пороговой энергии малоразмерного телескопа (близкого по параметрам к телескопу TAIGA-IACT) с камерой на SiPM OnSemi MicroFJ-60035 будет находится в интервале $0.28-0.76 \mathrm{TeV}$ в зависимости от используемого типа фильтра, триггера, алгоритма очистки изображения и специфики решаемой задачи (что определяет требования к приемлемому/допустимому уровню ошибок измеряемых величин и скорости ложного счета). Наиболее жесткие из рассмотренных целесообразных пороговых условий (т. е. условия, гарантирующие высокое качество изображений подавляющей доли регистрируемых событий) приводят к значениям пороговой энергии $0.76 \mathrm{TeV}$ при наблюдениях с использованием фильтра основного диапазона $(260-600 \mathrm{~nm})$ и $0.72 \mathrm{TeV}$ при использовании фильтра типа ZWB3 (270-400 nm). Оба эти значения примерно в два раза меньше пороговой энергии текущей конфигурации телескопа TAIGA-IACT с камерой на вакуумных фотоумножителях ХР1911, которая составляет около $\simeq 1.5 \mathrm{TeV}[16]$.

Результаты настоящей работы подтверждают предварительную оценку значений пороговой энергии наблюдений $(\sim 0.6 \mathrm{TeV}$ при использовании фильтра основного диапазона и $\sim 0.3 \mathrm{TeV}$ при использовании фильтра типа ZWB3), сделанную в [17], но не подтверждают предварительный вывод [17] о возможности значительного (с 0.6 до $0.3 \mathrm{TeV}$ ) понижения пороговой энергии при применении УФ-фильтра ZWB3 вместо фильтра основного диапазона. Тем не менее - и это представляется весьма существенным - ухудшения значения пороговой энергии при смене фильтра основного диапазона на УФ-фильтр с сохранением типа адаптивного триггера $\left\{n_{\mathrm{th}} ; N^{\text {th }}\right\}$ также не происходит (при той же интенсивности фона ночного неба). Это подтверждает перспективность использования УФ-фильтров типа ZWB3 для увеличения длительности рабочего цикла телескопа (за счет возможности более длительной работы в сумерках и в лунные ночи).

\section{Благодарности}

Проведенные исследования могут быть использованы, в частности, для модернизации УНУ „Астрофизический комплекс МГУ-ИГУ“, осуществляемой в рамках соглашения 13.УНУ.21.0007 между Министерством образования и науки России и Иркутским государственным университетом.

\section{Финансирование работы}

Авторы благодарят Российский научный фонд (РНФ) за поддержку в рамках проекта 19-72-20045.

\section{Конфликт интересов}

Авторы заявляют об отсутствии конфликта интересов.

\section{Список литературы}

[1] C.C. Hsu, D. Fink, J. Hose, R. Mirzoyan, O. Reimann, M. Shayduk, M. Teshima. Nuclear Instruments and Methods in Physics Research A, 610, 267 (2009).

[2] E. Gazda, T. Nguyen, N. Otte, G. Richards. J. Instrument., 11, 11015 (2016).

[3] G. Giavitto, T. Ashton, A. Balzer, D. Berge, F. Brun, Th. Chaminade, E. Delagnes, G. Fontaine, M. Füßling, B. Giebels, J.-F. Glicenstein, T. Gräaber, J. Hinton, A. Jahnke, S. Klepser, M. Kossatz, A. Kretzschmann, V. Lefranc, H. Leich, H. Lüdecke, I. Lypova, P. Manigot, V. Marandon, E. Moulin, M. de Naurois, P. Nayman, S. Ohm, M. Penno, D. Ross, D. Salek, M. Schade, Th. Schwab, R. Simoni, Ch. Stegmann, C. Steppa, J. Thornhill, F. Toussnel. Nuclear Instruments and Methods in Physics Research A, 876, 35 (2017).

[4] B.S. Acharya, I. Agudo, I. Al Samarai, R. Alfaro, J. Alfaro, C. Alispach, R.A. Batista, J.-P. Amans, E. Amato, G. Ambrosi, E. Antolini, L.A. Antonelli, C. Aramo, M. Araya, T. Armstrong, F. Arqueros, L. Arrabito, K. Asano, M. Ashley, M. Backes, C. Balazs, M. Balbo, O. Ballester, J. Ballet, A. Bamba, M. Barkov, U.B. de Almeida, J.A. Barrio, D. Bastieri, Y. Becherini, A. Belfiore, W. Benbow, D. Berge, E. Bernardini, M.G. Bernardini, M. Bernardos, K. Bernlöhr, B. Bertucci, B. Biasuzzi, C. Bigongiari, A. Biland, E. Bissaldi, J. Biteau, O. Blanch, J. Blazek, C. Boisson, J. Bolmont, G. Bonanno, A. Bonardi, C. Bonavolontá, G. Bonnoli, Z. Bosnjak, M. Böttcher, C. Braiding, J. Bregeon, A. Brill, A.M. Brown, P. Brun, G. Brunetti, T. Buanes, J. Buckley, V. Bugaev, R. Bühler, A. Bulgarelli, T. Bulik, M. Burton, A. Burtovoi, G. Busetto, R. Canestrari, M. Capalbi, F. Capitanio, A. Caproni, P. Caraveo, V. Cárdenas, C. Carlile, R. Carosi, E. Carquín, J. Carr, S. Casanova, E. Cascone, F. Catalani, O. Catalano, D. Cauz, M. Cerruti, P. Chadwick, S. Chaty, R.C.G. Chaves, A. Chen, X. Chen, M. Chernyakova, M. Chikawa, A. Christov, J. Chudoba, M. Cieślar, V. Coco, S. Colafrancesco, P. Colin, V. Conforti, J. Conrad, J.L. Contreras, J. Cortina, A. Costa, H. Costantini, G. Cotter, S. Covino, R. Crocker, J. Cuadra, O. Cuevas, P. Cumani, A. d'Aí, F. d'Ammando, P. d'Avanzo, D. d'Urso, M. Daniel, I. Davids, B. Dawson, F. Dazzi, A. de Angelis, R. de Cássia dos Anjos, G. de Cesare, A. de Franco, E.M. de Gouveia Dal Pino, I. de la Calle, R. de los Reyes Lopez, B. de Lotto, A. de Luca, M. de Lucia, M. de Naurois, E. de Oña Wilhelmi, F. de Palma, F. de Persio, V. de Souza, C. Deil, M. del Santo, C. Delgado, D. Della Volpe, T. Di Girolamo, F. Di Pierro, L. Di Venere, C. Díaz, C. Dib, S. Diebold, A. Djannati-Ataï, A. Domínguez, D.D. Prester, D. Dorner, M. Doro, H. Drass, D. Dravins, G. Dubus, V.V. Dwarkadas, J. Ebr, C. Eckner, K. Egberts, S. Einecke, T.R.N. Ekoume, D. Elsässer, J.-P. Ernenwein, C. Espinoza, C. Evoli, M. Fairbairn, D. Falceta-Goncalves, A. Falcone, G. Fasola, E. Fedorova, S. Fegan, M. Fernandez-Alonso, A. Fernández-Barral, G. Ferrand, M. Fesquet, M. Filipovic, V. Fioretti, G. Fontaine, M. Fornasa, L. Fortson, L.F. Coromina, C. Fruck, Y. Fujita, Y. Fukazawa, S. Funk, M. Füßling, S. Gabici, A. Gadola, Y. Gallant, B. Garcia, R. Garcia López, M. Garczarczyk, T. Gasparetto, M. Gaug, G. Giavitto, 
N. Giglietto, F. Giordano, E. Giro, M. Giroletti, A. Giuliani, J.F. Glicenstein, R. Gnatyk, N. Godinovic, P. Goldoni, G. GómezVargas, M.M. González, J.M. González, D. Götz, J. Graham, P. Grandi, J. Granot, A.J. Green, T. Greenshaw, S. Griffiths, S. Gunji, D. Hadasch, S. Hara, M.J. Hardcastle, T. Hassan, K. Hayashi, M. Hayashida, M. Heller, J.C. Helo, G. Hermann, J. Hinton, B. Hnatyk, W. Hofmann, J. Holder, D. Horan, J. Hörandel, D. Horns, P. Horvath, T. Hovatta, M. Hrabovsky, D. Hrupec, T.B. Humensky, M. Hütten, M. Iarlori, T. Inada, Y. Inome, S. Inoue, T. Inoue, Y. Inoue, F. Iocco, K. Ioka, M. Iori, K. Ishio Y. Iwamura M. Jamrozy P. Janecek D. Jankowsky, P. Jean, I. Jung-Richardt, J. Jurysek, P. Kaaret, S. Karkar, H. Katagiri, U. Katz, N. Kawanaka, D. Kazanas, B. Khélifi, D.B. Kieda, S. Kimeswenger, S. Kimura, S. Kisaka, J. Knapp, J. Knödlseder, B. Koch, K. Kohri, N. Komin, K. Kosack, M. Kraus, M. Krause, F. Krauß, H. Kubo, G. Kukec Mezek, H. Kuroda, J. Kushida, N. La Palombara, G. Lamanna, R.G. Lang, J. Lapington, O. Le Blanc, S. Leach, J.-P. Lees, J. Lefaucheur, Leigui de Oliveira, J.-P. Lenain, R. Lico, M. Limon, E. Lindfors, T. Lohse, S. Lombardi, F. Longo, M. López, R. López-Coto, F. Lucarelli, P.L. LuqueEscamilla, E. Lyard, M.C. Maccarone, G. Maier, P. Majumdar, G. Malaguti, D. Mandat, G. Maneva, M. Manganaro, S. Mangano, A. Marcowith, J. Marín, S. Markoff, J. Martí, P. Martin, M. Martínez, Gines Martinez Garcia, N. Masetti, S. Masuda, G. Maurin, N. Maxted, D. Mazin, C. Medina, A. Melandri, S. Mereghetti, M. Meyer, I.A. Minaya, N. Mirabal, R. Mirzoyan, A. Mitchell, T. Mizuno, R. Moderski, M. Mohammed, L. Mohrmann, T. Montaruli, A. Moralejo, D. Morcuende-Parrilla, K. Mori, G. Morlino, P. Morris, A. Morselli, E. Moulin, R. Mukherjee, C. Mundell, T. Murach, H. Muraishi, K. Murase, A. Nagai, S. Nagataki, T. Nagayoshi, T. Naito, T. Nakamori, Y. Nakamura, J. Niemiec, D. Nieto, M. Nikołajuk, K. Nishijima, K. Noda, D. Nosek, B. Novosyadlyj, S. Nozaki, P. O’Brien, L. Oakes, Y. Ohira, M. Ohishi, S. Ohm, N. Okazaki, A. Okumura, R.A. Ong, M. Orienti, R. Orito, J.P. Osborne, M. Ostrowski, N. Otte, I. Oya, M. Padovani, A. Paizis, M. Palatiello, M. Palatka, R. Paoletti, J.M. Paredes, G. Pareschi, R.D. Parsons, A. Péer, M. Pech, G. Pedaletti, M. Perri, M. Persic, A. Petrashyk, P. Petrucci, O. Petruk, B. Peyaud, M. Pfeifer, G. Piano, A. Pisarski, S. Pita, M. Pohl, M. Polo, D. Pozo, E. Prandini, J. Prast, G. Principe, D. Prokhorov, H. Prokoph, M. Prouza, G. Pühlhofer, M. Punch, S. Pürckhauer, F. Queiroz, A. Quirrenbach, S. Rainó, S. Razzaque, O. Reimer, A. Reimer, A. Reisenegger, M. Renaud, A.H. Rezaeian, W. Rhode, D. Ribeiro, M. Ribó, T. Richtler, J. Rico, F. Rieger, M. Riquelme, S. Rivoire, V. Rizi, J. Rodriguez, G. Rodriguez Fernandez, J.J. Rodríguez Vázquez, G. Rojas, P. Romano, G. Romeo, J. Rosado, A.C. Rovero, G. Rowell, B. Rudak, A. Rugliancich, C. Rulten, I. Sadeh, S. Safi-Harb, T. Saito, N. Sakaki, S. Sakurai, G. Salina, M. Sánchez-Conde, H. Sandaker, A. Sandoval, P. Sangiorgi, M. Sanguillon, H. Sano, M. Santander, S. Sarkar, K. Satalecka, F.G. Saturni, E.J. Schioppa, S. Schlenstedt, M. Schneider, H. Schoorlemmer, P. Schovanek, A. Schulz, F. Schussler, U. Schwanke, E. Sciacca, S. Scuderi, I. Seitenzahl, D. Semikoz, O. Sergijenko, M. Servillat, A. Shalchi, R.C. Shellard, L. Sidoli, H. Siejkowski, A. Sillanpää, G. Sironi, J. Sitarek, V. Sliusar, A. Slowikowska, H. Sol, A. Stamerra, S. Stanič, R. Starling, Ł. Stawarz, S. Stefanik, M. Stephan, T. Stolarczyk, G. Stratta, U. Straumann, T. Suomijarvi, A.D. Supanitsky, G. Tagliaferri, H. Tajima,
M. Tavani, F. Tavecchio, J.-P. Tavernet, K. Tayabaly, L.A. Tejedor, P. Temnikov, Y. Terada, R. Terrier, T. Terzic, M. Teshima, V. Testa, S. Thoudam, W. Tian, L. Tibaldo, M. Tluczykont, C.J. Todero Peixoto, F. Tokanai, J. Tomastik, D. Tonev, M. Tornikoski, D.F. Torres, E. Torresi, G. Tosti, N. Tothill, G. Tovmassian, P. Travnicek, C. Trichard, M. Trifoglio, I. Troyano Pujadas, S. Tsujimoto, G. Umana, V. Vagelli, F. Vagnetti, M. Valentino, P. Vallania, L. Valore, C. van Eldik, J. Vandenbroucke, G.S. Varner, G. Vasileiadis, V. Vassiliev, M. Vázquez Acosta, M. Vecchi, A. Vega, S. Vercellone, P. Veres, S. Vergani, V. Verzi, G.P. Vettolani, A. Viana, C. Vigorito, J. Villanueva, H. Voelk, A. Vollhardt, S. Vorobiov, M. Vrastil, T. Vuillaume, S.J. Wagner, R. Wagner, R. Walter, J.E. Ward, D. Warren, J.J. Watson, F. Werner, M. White, R. White, A. Wierzcholska, P. Wilcox, M. Will, D.A. Williams, R. Wischnewski, T. Yamamoto, R. Yamazaki, S. Yanagita, L. Yang, T. Yoshida, S. Yoshiike, T. Yoshikoshi, M. Zacharias, G. Zaharijas, L. Zampieri, F. Zandanel, R. Zanin, M. Zavrtanik, D. Zavrtanik, A.A. Zdziarski, A. Zech, H. Zechlin, V.I. Zhdanov, A. Ziegler, J. Zorn. The Cherenkov Telescope Array Consortium, Science with the Cherenkov Telescope Array. ArXive-prints (2017).

[5] А.М. Быков, Ф.А. Агаронян, А.М. Красильщиков, Е.Е. Холупенко, П.Н. Аруев, Д.А. Байко, А.А. Богданов, Г.И. Васильев, В.В. Забродский, С.В. Троицкий, Ю.В. Тубольцев, А.А. Кожберов, К.П. Левенфиш, Ю.В. Чичагов. ЖТФ, 87 (6), 803 (2017). [A.M. Bykov, F.A. Aharonian, A.M. Krassilchtchikov, E.E. Kholupenko, P.N. Aruev, D.A. Baiko, A.A. Bogdanov, G.I. Vasilyev, V.V. Zabrodskii, S.V. Troitsky, Y.V. Tuboltsev, A.A. Kozhberov, K.P. Levenfish, Y.V. Chichagov. Tech. Phys., 62, 819 (2017)]

[6] H. Anderhub, M. Backes, A. Biland, V. Boccone, I. Braun, T. Bretz, J. Buß, F. Cadoux, V. Commichau, L. Djambazov, D. Dorner, S. Einecke, D. Eisenacher, A. Gendotti, O. Grimm1, H. von Gunten, C. Haller, D. Hildebrand, U. Horisberger, B. Huber, K.-S. Kim, M.L. Knoetig, J.H. Köhne, T. Krähenbühl, B. Krumm, M. Lee, E. Lorenz, W. Lustermann, E. Lyard, K. Mannheim, M. Meharga, K. Meier, T. Montaruli, D. Neise, F. Nessi-Tedaldi, A.K. Overkemping, A. Paravac, F. Pauss, D. Renker, W. Rhode, M. Ribordy, U. Röser, J.-P. Stucki, J. Schneider, T. Steinbring, F. Temme, J. Thaele, S. Tobler, G. Viertel, P. Vogler, R. Walter, K. Warda, Q. Weitzel, M. Zänglein. J. Instrumentation, 8, 06008 (2013).

[7] G. Ambrosi, M. Ambrosio, C. Aramo, E. Bissaldi, A. Boiano, A. Bonavolontá, C. de Lisio, L. Di Venere, E. Fiandrini, N. Giglietto, F. Giordano, M. Ionica, V. Masone, R. Paoletti, V. Postolache, D. Simone, V. Vagelli, M. Valentino and CTA Consortium. Nuclear and Particle Physics Proceedings, 291293, 55 (2017).

[8] M. Mallamaci, B. Baibussinov, G. Busetto, D. Corti, A. De Angelis, F. Di Pierro, M. Doro, L. Lessio, M. Mariotti, R. Rando, E. Prandini, P. Vallania, C.F. Vigorito and CTA LST Project. Nuclear Instruments and Methods in Physics Research A, 936, 231 (2019).

[9] G. Ambrosi, M. Ambrosio, C. Aramo, B. Bertucci, E. Bissaldi, M. Bitossi, A. Boiano, C. Bonavolontá, M. Caprai, L. Consiglio, L. Di Venere, E. Fiandrini, N. Giglietto, F. Giordano, M. Ionica, F. Licciulli, S. Loporchio, V. Masone, F.R. Pantaleo, R. Paoletti, A. Rugliancich, L. Stiaccini, L. Tosti, V. Vagelli, M. Valentino and CTA SCT Project. Nuclear and Particle Physics Proceedings, 306-308, 37, (2019). 
[10] S. Lombardi, O. Catalano, S. Scuderi, L.A. Antonelli, G. Pareschi, E. Antolini, L. Arrabito, G. Bellassai, K. Bernlöhr, C. Bigongiari, B. Biondo, G. Bonanno, G. Bonnoli, G.M. Böttcher, J. Bregeon, P. Bruno, R. Canestrari, M. Capalbi, P. Caraveo, P. Conconi, V. Conforti, G. Contino, G. Cusumano, E. M. de Gouveia Dal Pino, A. Distefano, G. Farisato, C. Fermino, M. Fiorini, A. Frigo, S. Gallozzi, C. Gargano, S. Garozzo, F. Gianotti, S. Giarrusso, R. Gimenes, E. Giro, A. Grillo, D. Impiombato, S. Incorvaia, N. La Palombara, V. La Parola, G. La Rosa, G. Leto, F. Lucarelli, M.C. Maccarone, D. Marano, E. Martinetti, A. Micciché, R. Millul, T. Mineo, G. Nicotra, G. Occhipinti, I. Pagano, M. Perri, G. Romeo, F. Russo, F. Russo, B. Sacco, P. Sangiorgi, F.G. Saturni, A. Segreto, G. Sironi, G. Sottile, A. Stamerra, L. Stringhetti, G. Tagliaferri, M. Tavani, V. Testa, M.C. Timpanaro, G. Toso, G. Tosti, M. Trifoglio, G. Umana, S. Vercellone, R. Zanmar Sanchez, C. Arcaro, A. Bulgarelli, M. Cardillo, E. Cascone, A. Costa, A. D’Aí, F. D’Ammando, M. Del Santo, V. Fioretti, A. Lamastra, S. Mereghetti, F. Pintore, G. Rodeghiero, P. Romano, J. Schwarz, E. Sciacca, F.R. Vitello, A. Wolter. Astronomy and Astrophysics, 634, A22 (2020).

[11] C.B. Adams, R. Alfaro, G. Ambrosi, M. Ambrosio, C. Aramo, T. Arlen, P.I. Batista, W. Benbow, B. Bertucci, E. Bissaldi, J. Biteau, M. Bitossi, A. Boiano, C. Bonavolontá, R. Bose, A. Bouvier, A. Brill, A.M. Brown, J.H. Buckley, K. Byrum, R.A. Cameron, R. Canestrari, M. Capasso, M. Caprai, C.E. Covault, D. Depaoli, M. Errando, S. Fegan, Q. Feng, E. Fiandrini, G. Foote, P. Fortin, S. Funk, A. Furniss, F. Garfias, A. Gent, N. Giglietto, F. Giordano, E. Giro, M.M. González, V. Guarino, R. Halliday, O. Hervet, J. Holder, G. Hughes, T.B. Humensky, M. Ionica, A. Iriarte, W. Jin, C.A. Johnson, P. Kaaret, D. Kieda, B. Kim, A. Kuznetsov, J.S. Lapington, F. Licciulli, S. Loporchio, V. Masone, K. Meagher, T. Meures, B.A.W. Mode, S.A.I. Mognet, R. Mukherjee, T. Nguyen, D. Nieto, A. Okumura, N. Otte, N. La Palombara, F.R. Pantaleo, R. Paoletti, G. Pareschi, A. Petrashyk, F. Di Pierro, E. Pueschel, P.T. Reynolds, D. Ribeiro, G. Richards, E. Roache, D. Ross, J. Rousselle, A. Rugliancich, J. Ruíz-Díaz-Soto, M. Santander, S. Schlenstedt, M. Schneider, S. Scuderi, R. Shang, G. Sironi, B. Stevenson, L. Stiaccini, H. Tajima, L.P. Taylor, J. Thornhill, L. Tosti, G. Tovmassian, V. Vagelli, M. Valentino, J. Vandenbroucke, V.V. Vassiliev, L. Di Venere, S.P. Wakely, J.J. Watson, R. White, P. Wilcox, D.A. Williams, M. Wood, P. Yu, A. Zink. Astroparticle Physics, 128, 102562 (2021).

[12] M.L. Knoetig, A. Biland, T. Bretz, J. Buss, D. Dorner, S. Einecke, D. Eisenacher, D. Hildebrand, T. Krahenbuhl, W. Lustermann, K. Mannheim, K. Meier, D. Neise, A.K. Overkemping, A. Paravac, F. Pauss, W. Rhode, M. Ribordy, T. Steinbring, F. Temme, J. Thaele, P. Vogler, R. Walter, Q. Weitzel, M. Zanglein. FACTLong-Term Stability and Observations during Strong Moon Light. In International Cosmic Ray Conference, V. 33 of International Cosmic Ray Conference, 1132 (2013).

[13] E.E. Kholupenko, P.N. Aruev, D.A. Baiko, A.A. Bogdanov, G.I. Vasilyev, V.V. Zabrodskii, A.M. Krasil'shchikov, Y.V. Tuboltsev, Y.V. Chichagov. Physics of Atomic Nuclei, 79, 1542 (2016).

[14] L.A. Kuzmichev, I.I. Astapov, P.A. Bezyazeekov, V. Boreyko, A.N. Borodin, N.M. Budnev, R. Wischnewski, A.Y. Garmash, A.R. Gafarov, N.V. Gorbunov, V.M. Grebenyuk, O.A. Gress, T.I. Gress, A.A. Grinyuk, O.G. Grishin, A.N. Dyachok,
A.V. Zagorodnikov, V.L. Zurbanov, A.L. Ivanova, Y.A. Kazarina, N.N. Kalmykov, N.I. Karpov, V.V. Kindin, P.S. Kirilenko, S.N. Kiryuhin, V.A. Kozhin, R.P. Kokoulin, K.G. Kompaniets, E.E. Korosteleva, E.A. Kravchenko, M. Kunnas, A. Chiavassa, A.A. Lagutin, V.V. Lenok, B.K. Lubsandorzhiev, N.B. Lubsandorzhiev, R.R. Mirgazov, R. Mirzoyan, R.D. Monkhoev, R. Nachtigall, E.A. Osipova, M.I. Panasyuk, L.V. Pankov, A.L. Pakhorukov, A.A. Petrukhin, V.A. Poleschuk, M. Popesku, E.G. Popova, A. Porelli, E.B. Postnikov, V.V. Prosin, V.S. Ptuskin, A.A. Pushnin, R.I. Raikin, G.I. Rubtsov, Ya.I. Sagan, V.S. Samoliga, L.G. Sveshnikova, Yu.A. Semeney, A.Y.Sidorenkov, A.A. Silaev, A.A. Silaev, A.V. Skurikhin, M. Slunecka, A.V. Sokolov, V.P. Sulakov, V.A. Tabolenko, B.A. Tarashansky, L.G. Tkachev, A.V. Tkachenko, M. Tluczykont, O.L. Fedorov, A.D. Horns, C. Spiering, A.Sh.M. Elshoukrofy, I.I. Yashin. Physics of Atomic Nuclei, 81 (4), (2018).

[15] ON Semiconductor. J-Series SiPM Sensors. Silicon Photomultipliers(SiPM), High PDE and Timing Resolution Sensors in a TSV Package; https://www.onsemi.com/pub/Collateral/MICROJ-SERIESD.PDF, 2017.

[16] Л.А. Кузьмичев. Частное сообщение, 2020.

[17] Е.Е. Холупенко, А.М. Красильщиков, Д.В. Бадмаев, А.А. Богданов, Ю.В. Тубольцев, Ю.В. Чичагов, А.С. Антонов, Д.О. Кулешов, Е.М. Хилькевич. ЖТФ, 87 (6), 925 (2020). [E.E. Kholupenko, A.M. Krassilchtchikov, D.V. Badmaev, A.A.Bogdanov, Yu.V. Tuboltsev, Yu.V. Chichagov, A.S. Antonov, D.O. Kuleshov, E.M. Khil'kevich. Tech. Phys., 65 (6), 886 (2020)]

[18] C. Leinert, S. Bowyer, L.K. Haikala, M.S. Hanner, M.G. Hauser, A.-C. Levasseur-Regourd, I. Mann, K. Mattila, W.T. Reach, W. Schlosser, H.J. Staude, G.N. Toller, J.L. Weiland, J.L. Weinberg, A.N. Witt. Astronomy and Astrophysics Supplement, 127, 1 (1998).

[19] C.R. Benn, S.L. Ellison. New Astronomy Reviews, 42 (6-8), 503 (1998).

[20] M. Chantell, C.W. Akerlof, J. Buckley, D.A. CarterLewis, M.F. Cawley, V. Connaughton, D.J. Fegan, P. Fleury, J. Gaidos, A.M. Hillas, R.C. Lamb, E. Pare, H.J. Rose, A.C. Rovero, X. Sarazin, G. Sembroski, M.S. Schubnell, M. Urban, T.C. Weekes, C. Wilson. Gamma-RayObservations in Moonlight with the Whipple Atmospheric Cherenkov Hybrid Camera. International Cosmic Ray Conference, 2, 544 (1995).

[21] M.C. Chantell, X. Sarazin, P. Fleury, K. Harris, A. Kerrick, E. Paré, M. Punch, M. Urban, G. Vacanti, T.C. Weekes. The Use of a UV Camera in the Atmospheric Cerenkov Technique. International Cosmic Ray Conference, 2, 560 (1995).

[22] D. Guberman, J. Cortina, R. García, J. Herrera, M. Manganaro, A. Moralejo, J. Rico, M. Will and MAGIC Collaboration. UsingUV-pass filters for bright Moon observations with MAGIC. In 34th International Cosmic Ray Conference (ICRC2015), 34, 1237 (2015).

[23] M.L. Ahnen, S. Ansoldi, L.A. Antonelli, C. Arcaro, A. Babić, B. Banerjee, P. Bangale, U. Barres de Almeida, J.A. Barrio, J. Becerra González, W. Bednarek, E. Bernardini, A. Berti, W. Bhattacharyya, B. Biasuzzi, A. Biland, O. Blanch, S. Bonnefoy, G. Bonnoli, R. Carosi, A. Carosi, A. Chatterjee, P. Colin, E. Colombo, J.L. Contreras, J. Cortina, S. Covino, P. Cumani, P. Da Vela, F. Dazzi, A. De Angelis, B. De Lotto, E. de Oña Wilhelmi, F. Di Pierro, M. Doert, A. Domínguez, 
D. Dominis Prester, D. Dorner, M. Doro, S. Einecke, D. Eisenacher Glawion, D. Elsaesser, M. Engelkemeier, V. Fallah Ramazani, A. Fernández-Barral, D. Fidalgo, M.V. Fonseca, L. Font, C. Fruck, D. Galindo, R.J. García Löpez, M. Garczarczyk, M. Gaug, P. Giammaria, N. Godinović, D. Gora, S. Griffiths, D. Guberman, D. Hadasch, A. Hahn, T. Hassan, M. Hayashida, J. Herrera, J. Hose, D. Hrupec, G. Hughes, K. Ishio, Y. Konno, H. Kubo, J. Kushida, D. Kuveždić, D. Lelas, E. Lindfors, S. Lombardi, F. Longo, M. Löpez, C. Maggio, P. Majumdar, M. Makariev, G. Maneva, M. Manganaro, K. Mannheim, L. Maraschi, M. Mariotti, M. Martínez, D. Mazin, U. Menzel, M. Minev, R. Mirzoyan, A. Moralejo, V. Moreno, E. Moretti, V. Neustroev, A. Niedzwiecki, M. Nievas Rosillo, K. Nilsson, D. Ninci, K. Nishijima, K. Noda, L. Nogués, S. Paiano, J. Palacio, D. Paneque, R. Paoletti, J.M. Paredes, X. Paredes-Fortuny, G. Pedaletti, M. Peresano, L. Perri, M. Persic, P.G. Prada Moroni, E. Prandini, I. Puljak, J.R. Garcia, I. Reichardt, W. Rhode, M. Ribó, J. Rico, A. Rugliancich, T. Saito, K. Satalecka, S. Schroeder, T. Schweizer, A. Sillanpää, J. Sitarek, I. Šnidarić, D. Sobczynska, A. Stamerra, M. Strzys, T. Surić, L. Takalo, F. Tavecchio, P. Temnikov, T. Terzić, D. Tescaro, M. Teshima, D.F. Torres, N. Torres-Albá, A. Treves, G. Vanzo, M. Vazquez Acosta, I. Vovk, J.E. Ward, M. Will, D. Zarić. Astroparticle Physics, 94, 29 (2017).

[24] S. Griffin and VERITAS Collaboration. VERITAS Observations under Bright Moonlight. In 34th International Cosmic Ray Conference(ICRC2015), 34, 989 (2015).

[25] A.M. Hillas. Cerenkov Light Images of EAS Produced by Primary Gamma Rays and by Nuclei. In 10th International Cosmic Ray Conference (ICRC19), 3, 445 (1985).

[26] Y.L. Zyskin, B.M. Vladimirsky, Y.I. Neshpor, A.A. Stepanov, V.P. Fomin, V.G. Shitov. The Investigation of Cerenkov Flashesin Ultraviolet Spectral Region. International Cosmic Ray Conference, 2, 342 (1987).

[27] Y.L. Neshpor, N.N. Chalenko, A.A. Stepanian, O.R. Kalekin, N.A. Jogolev, V.P Fomin, V.G. Shitov. Astronomy Reports, 45,249 (2001).

[28] M.A. Rahman, P.N. Bhat, B.S. Acharya, V.R. Chitnis, P. Majumdar, P.R. Vishwanath. Experimental Astronomy, 11, 113 (2001).

[29] Е.Е. Холупенко, А.М. Быков, Ф.А. Агаронян, Г.И. Васильев, А.М. Красильщиков, П.Н. Аруев, В.В. Забродский, А.В. Николаев. ЖТФ, 88 (11), 1655 (2018). [E.E. Kholupenko, A.M. Bykov, F.A. Aharonyan, G.I. Vasiliev, A.M. Krassilchtchikov, P.N. Aruev, V.V. Zabrodskii, A.V. Nikolaev. Tech. Phys., 63, 1603 (2018)]

[30] D. Heck, J. Knapp, J.N. Capdevielle, G. Schatz, T. Thouw. CORSIKA: a Monte Carlo code to simulate extensive air showers. (Forschungszentrum Karlsruhe $\mathrm{GmbH}$, Karlsruhe, Germany, TIB Hannover, D-30167 Hannover, Germany, 1998).

[31] E. Thébault, Ch.C. Finlay, C.D. Beggan, P. Alken, J. Aubert, O. Barrois, F. Bertrand, T. Bondar, A. Boness, L. Brocco, E. Canet, A. Chambodut, A. Chulliat, P. Coïsson, F. Civet, A. Du, A. Fournier, I. Fratter, N. Gillet, B. Hamilton, M. Hamoudi, G. Hulot, Th. Jager, M. Korte, W. Kuang, X. Lalanne, B. Langlais, J.-M. Léger, V. Lesur, F.J. Lowes, S. Macmillan, M. Mandea, Ch. Manoj, S. Maus, N. Olsen, V. Petrov, V. Ridley, M. Rother, T.J. Sabaka, D. Saturnino, R. Schachtschneider, O. Sirol, A. Tangborn, A. Thomson, L. Tøffner-Clausen, P. Vigneron, I. Wardinski, T. Zvereva.
International Geomagnetic Reference Field: the 12th generation. Earth, Planets, and Space, 67, 79 (2015).

[32] Р.В. Васильев, О.А. Гресс, Е.Е. Коростелева, Л.А. Кузьмичев, Б.К. Лубсандоржиев, А.И. Панфилов, Ю.В. Парфенов, Л.В. Паньков, В.В. Просин, Б.А. Шайбонов, К. Шпиринг, Т. Шмидт, Д.В. Чернов, И.В. Яшин. Приборы и техника эксперимента, 2, 17 (2009).

[33] F.X. Kneizys, L.W. Abreu, G.P. Anderson, J.H. Chetwynd, E.P. Shettle, A. Berk, L.S. Bernstein, D.C. Robertson, P. Acharya, L.S. Rothman, J.E.A. Selby, W.O. Gallery, S.A. Clough. The MODTRAN 2/3 report and LOWTRAN 7 model prepared for Phillips Laboratory, Geophysics Directorate, Hanscom AFB, MA 01731, U.S.A.; prepared by: Ontar Corporation, 9 Village Way, North Andover, MA 01845, 1996.

[34] C. Köhler, G. Hermann, W. Hofmann, A. Konopelko, A. Plyasheshnikov. Astroparticle Physics, 6 (1), 77 (1996).

[35] Г.И. Васильев, Е.Е. Холупенко, Д.А. Байко, А.М. Быков, А.М. Красильщиков, Г.Г. Павлов. Научно-технические ведомости СПбГПУ. Физико-математические науки, 134 (4), 79 (2011).

[36] Г.И. Васильев, Е.Е. Холупенко, С.Н. Яблоков, Д.А. Байко, А.М. Быков, А.М. Красильщиков, Г.Г. Павлов. Научнотехнические ведомости СПбГПУ. Физико-математические науки, 153 (3), 191 (2012).

[37] GEANT4. A simulation toolkit. https://geant4.web.cern.ch/ node $/ 1$.

[38] J. Allison, K. Amako, J. Apostolakis, P. Arce, M. Asai, T. Aso, E. Bagli, A. Bagulya, S. Banerjee, G. Barrand, B.R. Beck, A.G. Bogdanov, D. Brandt, J.M.C. Brown, H. Burkhardt, Ph. Canal, D. Cano-Ott, S. Chauvie, K. Cho, G.A.P. Cirrone, G. Cooperman, M.A. Cortés-Giraldo, G. Cosmo, G. Cuttone, G. Depaola, L. Desorgher, X. Dong, A. Dotti, V.D. Elvira, G. Folger, Z. Francis, A. Galoyan, L. Garnier, M. Gayer, K.L. Genser, V.M. Grichine, S. Guatelli, P. Guéye, P. Gumplinger, A.S. Howard, I. Hřivnáčová, S. Hwang, S. Incerti, A. Ivanchenko, V.N. Ivanchenko, F.W. Jones, S.Y. Jun, P. Kaitaniemi, N. Karakatsanis, M. Karamitrosi, M. Kelsey, A. Kimura, T. Koi, H. Kurashige, A. Lechner, S.B. Lee, F. Longo, M. Maire, D. Mancusi, A. Mantero, E. Mendoza, B. Morgan, K. Murakami, T. Nikitina, L. Pandola, P. Paprocki, J. Perl, I. Petrović, M.G. Pia, W. Pokorski, J.M. Quesada, M. Raine, M.A. Reis, A. Ribon, A. Ristić Fira, F. Romano, G. Russo, G. Santin, T. Sasaki, D. Sawkey, J.I. Shin, I.I. Strakovsky, A. Taborda, S. Tanaka, B. Tomé, T. Toshito, H.N. Tran, P.R. Truscott, L. Urban, V. Uzhinsky, J.M. Verbeke, M. Verderi, B.L.Wendt, H. Wenzel, D.H. Wright, D.M. Wright, T. Yamashita, J. Yarba, H. Yoshida. Nuclear Instrumentsand Methods in Physics Research A, 835, 186 (2016).

[39] D. Heck. Private communication, April 2020.

[40] A.V. Mikhalev, I.V Medvedeva, A.B. Beletsky, E.S. Kazimirovsky. J. Atmospheric and Solar-Terrestrial Physics, 63, 865 (2001).

[41] А.В. Михалев, И.В Медведева. Оптика атмосферы и океана, 15, 993 (2002).

[42] R. Mirzoyan, E. Lorenz. Measurement ofthe night sky light background at La Palma. Internal report of the HEGRA collaboration. MPI-PhE/94-35, 1994.

[43] N. Lubsandorzhiev, I. Astapov, P. Bezyazeekov, V. Boreyko, A. Borodin, M. Brueckner, N. Budnev, A. Chiavassa, A. Dyachok, O. Fedorov, A. Gafarov, A. Garmash, N. Gorbunov, V. Grebenyuk, O. Gress, T. Gress, O. Grishin, 
A. Grinyuk, D. Horns, A. Igoshin, A. Ivanova, N. Kalmykov, Y. Kazarina, V. Kindin, P. Kirilenko, S. Kiryuhin, R. Kokoulin, K. Kompaniets, E. Korosteleva, V. Kozhin, E. Kravchenko, M. Kunnas, L. Kuzmichev, Y. Lemeshev, V. Lenok, B. Lubsandorzhiev, R. Mirgazov, R. Mirzoyan, R. Monkhoev, R. Nachtigall, E. Osipova, A. Pakhorukov, M.I. Panasyuk, L. Pankov, A. Petrukhin, V. Poleschuk, E. Popescu, E. Popova, A. Porelli, E. Postnikov, V. Prosin, V. Ptuskin, E. Rjabov, G. Rubtsov, A. Pushnin, Y. Sagan, B. Sabirov, V. Samoliga, Y. Semeney, A. Silaev, A. Silaev (junior), A. Sidorenkov, A. Skurikhin, V. Slunecka, A.V. Sokolov, C. Spiering, L. Sveshnikova, V. Tabolenko, B. Tarashansky, A. Tkachenko, L. Tkachev, M. Tluczykont, R. Wischnewski, A. Zagorodnikov, D. Zhurov, V. Zurbanov, I. Yashin. Camera of the first TAIGAIACT: construction and calibration. In 35th International Cosmic Ray Conference (ICRC2017), volume 301 of International Cosmic Ray Conference, page 757, Jan 2017.

[44] D. Berge. Development of an Algorithm for the Shower Reconstruction with the H.E.S.S. Telescope System. Dissertation, Humboldt-Universitat zu Berlin, 2002.

[45] M.Sc. Tulun Ergin. The Energy Spectrum of Very High Energy Gamma Rays from the Crab Nebula as Measured by the H.E.S.S. Array. dissertation, Humboldt-Universitat zu Berlin, 2005.

[46] R. Holzlöhner, J. Kosmalski, S. Guisard. Stray light and thermal self-emission minimization at the ELT. In Groundbased and Airborne Telescopes VII, Society of Photo-Optical Instrumentation Engineers (SPIE) Conference Series, 10700, 107000I (2018).

[47] E. Roache, R. Irvin, J.S. Perkins, K. Harris, A. Falcone, J. Finley, T. Weeks. Mirror Facets for the VERITAS Telescopes. In International Cosmic Ray Conference, 3, 1397 (2008).

[48] В.А. Зверев, Е.В. Кривопустова, Т.В. Точилина. Оптические материалы. Часть 2. Учебное пособие для конструкторов оптических систем. (Санкт-петербургский национальный исследовательский университет информационных технологий, механики и оптики, СпБ, 2013)

[49] F. Hénault, P.-O. Petrucci, L. Jocou, B. Khélifi, P. Manigot, S. Hormigos, J. Knödlseder, J.-F. Olive, P. Jean, M. Punch. Design of light concentrators for Cherenkov telescope observatories. In Nonimaging Optics: Efficient Design for Illumination and Solar Concentration X, Society of PhotoOptical Instrumentation Engineers (SPIE), Conference Series, 8834, 883405 (2013).

[50] А.С. Антонов, А.А. Богданов, А.М. Красильщиков, Е.Е. Холупенко. ЖТФ, 91 (11), 1 (2021).

[51] C. Berat, S. Bottai, D. De Marco, S. Moreggia, D. Naumov, M. Pallavicini, R. Pesce, A. Petrolini, A. Stutz, E. Taddei, A. Thea. Astroparticle Physics, 33, 221 (2010).

[52] HEGRA Collaboration, A. Konopelko, M. Hemberger, F. Aharonian, A. Daum, W. Hofmann, C. Köhler, H. Krawczynski, H.J. Völk, A. Akhperjanian, J. Barrio, K. Bernlöhr, H. Bojahr, J. Contreras, J. Cortina, T. Deckers, S. Denninghoff, J. Fernandez, V. Fonseca, J. Gonzalez, V. Haustein, G. Heinzelmann, G. Hermann, M. Hess, A. Heusler, H. Hohl, I. Holl, D. Horns, R. Kankanian, M. Kestel, J. Kettler, O. Kirstein, H. Kornmayer, D. Kranich, H. Lampeitl, A. Lindner, E. Lorenz, N. Magnussen, H. Meyer, R. Mirzoyan, H. Möller, A. Moralejo, L. Padilla, M. Panter, D. Petry, R. Plaga, A. Plyasheshnikov, J. Prahl, C. Prosch, G. Pühlhofer, G. Rauterberg, W. Rhode, A. Röhring, V. Sahakian, M. Samorski, J. Sanchez, D. Schmele,
F. Schroeder, W. Stamm, B. Wiebel-Sooth, C.A. Wiedner, M. Willmer, H. Wirth. Astroparticle Physics, $10(4), 275$ (1999).

[53] S. Schlenker. Simulation Study of the H.E.S.S. Single Telescope Trigger Rate. Dissertation, Humboldt-Universitat zu Berlin, 2001.

[54] S. Funk. Hadron Suppression Using Wavelet Transformations for the H.E.S.S. Telescope System. Dissertation (Humboldt-Universitat zu, Berlin, 2002).

[55] F. Aharonian, A. Akhperjanian, M. Beilicke, K. Bernlöhr, H.G. Börst, H. Bojahr, O Bolz, T. Coarasa, J.L. Contreras, J. Cortina, S. Denninghoff, M.V. Fonseca, M. Girma, N. Götting, G. Heinzelmann, G. Hermann, A. Heusler, W. Hofmann, D. Horns, I. Jung, R. Kankanyan, M. Kestel, A. Kohnle, A. Konopelko, D. Kranich, H. Lampeitl, M. Lopez, E. Lorenz, F. Lucarelli, O. Mang, D. Mazin, H. Meyer, R. Mirzoyan, A. Moralejo, E. Oña-Wilhelmi, M. Panter, A. Plyasheshnikov, G. Pühlhofer, R. de los Reyes, W. Rhode, J. Ripken, G. Rowell, V. Sahakian, M. Samorski, M. Schilling, M. Siems, D. Sobzynska, W. Stamm, M. Tluczykont, V. Vitale, H.J. Völk, C.A. Wiedner, W. Wittek. The Astrophysical J., 614 (2), 897 (2004).

[56] Описание космического гамма-источник Краб в он-лайн каталоге TeVCat, tevcat.uchicago.edu.http://tevcat.uchicago. edu $/$ ? mode $=1 \&$ showsrc $=74$.

[57] F. Aharonian, A. Akhperjanian, M. Beilicke, K. Bernlöhr, H.G. Börst, H. Bojahr, O. Bolz, T. Coarasa, J.L. Contreras, J. Cortina, S. Denninghoff, V. Fonseca, M. Girma, N. Götting, G. Heinzelmann, G. Hermann, A. Heusler, W. Hofmann, D. Horns, I. Jung, R. Kankanyan, M. Kestel, A. Konopelko, H. Kornmeyer, D. Kranich, H. Lampeitl, M. Lopez, E. Lorenz, F. Lucarelli, O. Mang, D. Mazin, H. Meyer, R. Mirzoyan, A. Moralejo, E. Ona-Wilhelmi, M. Panter, A. Plyasheshnikov, G. Pühlhofer, R. de los Reyes, W. Rhode, J. Ripken, G. Rowell, V. Sahakian, M. Samorski, M. Schilling, M. Siems, D. Sobzynska, W. Stamm, M. Tluczykont, V. Vitale, H.J. Völk, C.A. Wiedner, W. Wittek. Astronomy and Astrophysics, 421, 529 (2004).

[58] S. Funk, G. Hermann, J. Hinton, D. Berge, K. Bernlöhr, W. Hofmann, P. Nayman, F. Toussenel, P. Vincent. Astroparticle Physics, 22 (3-4), 285 (2004).

[59] A.E. Chudakov, V.L. Dadykin, V.I. Zatsepin, N.M. Nesterova. On the High Energy Photons from Local Sources. In International Cosmic Ray Conference, 4, 199 (1963).

[60] J.V. Jelley, N.A. Porter. Quart. J. Roy. Astron. Soc., 4, 275 (1963)

[61] W.N. Charman, J.H. Fruin, J.V. Jelley, D.J. Fegan, D.M. Jennings, E.P. O’Mongain, N.A. Porter, G.M. White. Searches for High Energy Gamma-rays from Pulsars Using a Fast Night Sky Čerenkov Detector. In International Cosmic Ray Conference, 1, 59 (1970).

[62] K. Greisen. Progress in Cosmic Ray Physics (Amsterdam, North-Holland, 1956), v. 3.

[63] J. Linsley. GIL: a Formula for EAS Longitudinal Profiles. International Cosmic Ray Conference, 2, 502 (2001).

[64] H.J. Völk, K. Bernlöhr. Experimental Astronomy, 25 (1-3), 173 (2009).

[65] A.A. Bogdanov, E.E. Kholupenko, Yu.V. Tuboltsev, Yu.V. Chichagov. Latvian J. Physicsand Technical Sciences, 57 (1-2), 13 (2020). 
[66] V.L. Manomenova, E.B. Rudneva, A.É. Voloshin, L.V. Soboleva, A.B. Vasil'ev, B.V. McHedlishvili. Crystallography Reports, 50 (5), 877 (2005).

[67] М.А. Забудько. СПЕЦИФИКАЦИЯ. УФ светофильтр SP 305 (ООО Фотооптик, 2016)

[68] O.R. Kalekin, Y.I. Neshpor, A.A. Stepanyan, Y.L. Zyskin, A.P. Kornienko, B.M. Vladimirskii, V.P. Fomin, V.G. Shitov. Astronomy Lett., 21, 163 (1995).

[69] Photonis, imaging sensors. XP1911 Photomultiplier Tubes, Product Specification, 1999. 